Article

\title{
Optimized Procedure to Schedule Physicians in an Intensive Care Unit: A Case Study
}

\author{
Lotfi Hidri ${ }^{1, *}$, Achraf Gazdar ${ }^{2}$ and Mohammed M. Mabkhot ${ }^{1}$ \\ 1 Industrial Engineering Department, College of Engineering, King Saud University, P.O. Box 800, \\ Riyadh 11421, Saudi Arabia; mmabkhot@ksu.edu.sa \\ 2 Software Engineering Department, College of Computer and Information Science, King Saud University, \\ P.O. Box 800, Riyadh 11421, Saudi Arabia; agazdar@ksu.edu.sa \\ * Correspondence: lhidri@ksu.edu.sa
}

Received: 19 September 2020; Accepted: 3 November 2020; Published: 6 November 2020

\begin{abstract}
Hospitals are facing an important financial pressure due to the increasing of the operating costs. Indeed, the growth for the hospitals' services demand causes a rising in the number of required qualified personnel. Enlarging the personnel number increases dramatically the fixed total cost. Based on some studies, $50 \%$ of operating costs in US hospitals are allocated to healthcare personnel. Therefore, reducing these types of costs without damaging the service quality becomes a priority and an obligation. In this context, several studies focused on minimizing the total cost by producing optimal or near optimal schedules for nurses and physicians. In this paper, a real-life physicians scheduling problem with cost minimization is addressed. This problem is encountered in an Intensive Care Unit (ICU) where the current schedule is manually produced. The manual schedule is generating a highly unbalanced load within physicians in addition to a high cost overtime. The manual schedule preparation is a time consuming procedure. The main objective of this work is to propose a procedure that systematically produces an optimal schedule. This optimal schedule minimizes the total overtime within a short time and should satisfies the faced constraints. The studied problem is mathematically formulated as an integer linear program. The constraints are real, hard, and some of them are non-classical ones (compared to the existing literature). The obtained mathematical model is solved using a state-of-the-art software. Experimental tests on real data have shown the performance of the proposed procedure. Indeed, the new optimal schedules reduce the total overtime by up to $69 \%$. In addition, a more balanced workload for physicians is obtained and several physician preferences are now satisfied.
\end{abstract}

Keywords: integer linear programming; Intensive Care Unit; physicians scheduling; optimal schedule

\section{Introduction}

Intensive Care Units (ICU) are departments in hospitals where critical cases receive intensive treatments to save their lives and to limit damages. These critical cases include burned persons, stop beating heart persons, car accident survivals, and other severe cases. Usually, physicians in ICU are working under high mental and physical stresses. This is because the ICU physicians are dealing with critical and serious cases of patients facing death. In this context, physicians are performing cardiopulmonary resuscitation, heart massage, or other special treatments according to received patients. These actions may last a long while and might be repeated several times per shift. On the other hand, these physicians have to deal directly with shocked relatives of patients.

The workload is usually exceeding the ICU capacity, and physicians are requested to work more than their scheduled shifts. Therefore, sleep deprivation is observed among physicians with all the consequences. This is due mainly to the shortage of medical personnel (nurses and physicians mainly). 
A recent study in Germany showed that, on average, 2.5 full time physician positions are vacant in each hospital [1]. This shortage forces the hospitals' managers to find effective procedures for scheduling the available personnel efficiently. During the elaboration of these procedures, the managers should take into account with a lot of attention the incurred medical personnel costs. Indeed, these costs represent almost half of the operating costs in hospitals [2].

Developing efficient procedures that reduce physicians costs while keeping the same service quality is among the main concerns of the hospitals' managers. This is crucial for the survival of the hospitals. In this context, under-staffing has negative consequences for the management of hospitals. Indeed, the under-staffing reduces the services quality [3], augments the queues length and waiting times [4]. This extends the stay of the physicians in the hospitals and increases their shift duration [5].

During the preparation of schedules in an ICU, the physicians preferences should be taken into account as much as possible. Indeed, several studies were conducted and demonstrated the strong impact of shift scheduling on the psychological and social conditions for physicians [6,7]. In this context, medical errors were reported because of the sleep deprivation [8]. On the other hand, the survival of hospitals depends enormously on reducing the personnel total costs [2]. Balancing between physicians' preferences and total costs is a hard task that requires extensive expertise.

The physician scheduling belongs to a larger field which is the personnel scheduling. This kind of scheduling consists of the assignment of personnel to shifts taking into account regulations, preferences, skills, required staffing, and several other requirements. The personnel scheduling is well studied in the literature and most of researches focused on particular real-world problems. The physician scheduling is presenting some particular characteristics that distinguish it from other personnel scheduling problems. Indeed, physicians present the main valuable and expensive resource in the hospitals [9]. In addition, the qualified physicians are difficult to be replaced due to their experience and expertise [10]. Therefore, managers take into account seriously the physicians' preferences and try their best to provide them with the best feasible schedules.

Another specific aspect impacting the physicians scheduling is the high volatility of the services' demand. Therefore, the workload distribution in hospitals is uncertain [11], and impacts the design of the shifts. Consequently, considering the flexibility of shifts while preparing the schedules is highly desirable and might have a positive impact. In this context, managers take into account uncertainty in healthcare facilities [12,13].

In this work, a case study of physician scheduling problem in an ICU is considered. In this ICU, a team is charged with the preparation of a monthly physician schedule. This is due to the monthly variability of the number of assigned physicians. The entire work of this team is manually performed with all the negative consequences. The long duration of the schedule preparation (more than two weeks) and large number of contradictions are among these negative consequences. In addition, the produced schedules present a lot of unbalanced workload and an excessive overtime. Furthermore, the physicians are always complaining and their preferences are not fully taken into account. In order to cope with these drawbacks, a computerized solution is a proposed solution. This solution is modeling the studied problem using an integer linear programming formulation. This formulation includes several non-classical (not encountered in the literature) and hard constraints requiring an additional effort during the modeling phase.

Converting the constraints, preferences, and needs into a mathematical model requires particular attention. Indeed, the selection of the decision variables and the way of expressing these needs, in terms of constraints, have a major impact on the complexity of the mathematical model. The more the mathematical model is complex, the more the resolution becomes hard. In this study, and in addition to classical constraints and preferences (that appeared in previous studies), new ones are taken into account. These new constraints (needs) contain the multidisciplinary ICU (more than one building with different specialties), two consecutive days as days off, working in the same team during the planning horizon (rigid constraint), and others. These needs are increasing dramatically the 
complexity of the proposed mathematical model by increasing the number of decision variables and the number of constraints.

The remainder of this paper is organized as follows. In Section 2, a literature review on the physicians scheduling is presented. In Section 3, the ICU department is introduced and the studied problem is defined. An integer linear programming formulation, modeling the addressed problem is proposed in Section 4. Experimental study is conducted on real data and the related results are presented in Section 5. Finally, a conclusion presenting future research direction on related topics is exhibited in Section 6.

\section{Literature Review}

The personnel scheduling problems were intensively studied in the literature. In this context, authors in [14] provided a detailed literature review on modeling these problem. In [15], an exhaustive literature review on personnel rostering is presented. Authors in [16] presented a detailed literature review of the personnel planning. A literature review on personnel planning with different level of skills, was provided in [17].

The majority of the proposed researches on healthcare personnel scheduling focused on the nurse scheduling problem. A detailed literature review on this topic is presented in [18,19]. A literature review for the nurses turnover topic is presented in [20]. In [21], authors presented an exhaustive literature review on the acute care staffing of nurses. The personnel scheduling in the operating rooms is investigated in [22], where a detailed literature review is provided.

The physician scheduling problem presents several differences compared to the nurse scheduling problem in terms of preferences, requirements, and specialties. A detailed literature review for physician scheduling problem until the end of 2016 is presented in [23]. Consequently, the presented literature review in this paper is limited to the related works from the beginning of 2017. In this context, [24] addressed a case study of physician scheduling problem arising in a USA hospital. This problem was modeled using a mathematical model. This model is used to solve optimally small size instances. In addition, a branch-and-cut algorithm was proposed to solve the studied problem for medium and large size instances. The proposed procedures were assessed over a real data test problems.

Authors in [25] investigated the problem of gynecologist scheduling. This problem arose in Jeroen Bosch Hospital, where the current schedule is facing an unbalanced number of outpatients over the weekdays. In order to solve this problem, a Mixed Integer Linear Program and two heuristics were proposed. Preliminary results on realistic data were satisfactory and promising. In [26], authors studied the non-operating room anesthesia workflow and its impact in physician scheduling. For that aim, some metrics were adopted to evaluate the efficiency of the non-operating room anesthesia.

The problem of duty rostering for physicians at an orthopedics and trauma surgery department was addressed in [27]. This problem real-life problem was formulated as an integer programming model. This integer programming model was assessed over real data and preliminary results showed its performance compared to a manually generated schedule.

In [28], the Lean Six Sigma methodology was used to evaluate the lead time in Interventional Radiology Department and its impact on physician scheduling. This study was conducted in order to reduce the total process time. Authors in [29] studied the problem of residents scheduling problem. An iterative procedure is proposed, where each iteration consists on solving an integer linear program. Computational results on real world data from the University of Michigan Pediatric Emergency Department were presented.

The work in [30] addressed the problem of clinic-doctor assignment problem (or the coordinated lab-clinics problem). In this problem, the outpatient is requested to perform some medical tests just before meeting physician, in order to get accurate results. The coordination between these two procedures is a hard task. In order to solve the studied problem, a mixed integer linear programming formulation was proposed. This approach is valid to solve small size problems. Consequently, 
a heuristic based on a branch-and-price algorithm was proposed. Experimental results conducted on real world data shows the efficiency of the proposed approach.

In [31], authors introduced a new way to deal with logistics in hospitals. The personnel scheduling problem was among the treated topics in the latter work. The problem was partially modeled using a mixed-integer linear program. In addition, an exact procedure based on column generation was proposed to find an optimal solution. The proposed procedure was tested over a benchmark data and the obtained results were promising.

In [32], authors addressed the problem of surgery operating rooms scheduling problem under stochastic considerations. These stochastic considerations are the duration of the surgery and the arrival time of patients. A dynamic scheduling approach, which is the drum-buffer-rope (DBR), was proposed. Monte Carlo simulation was used to assess the proposed approach.

The work in [33] addressed the problem of physician scheduling. The authors introduced some indicators to assess physicians satisfaction. These indicators allow the planer (scheduler) to know about how much the preferences of physicians are fulfilled. The problem is modeled as mixed integer linear program. An experimental study is carry out on a real world data retrieved from a German university hospital and the obtained results are satisfactory.

Authors in [34] studied the problem of physician scheduling in a cancer treatment center. The objective is to align the produced schedule with the patient flow and consequently decreasing the treatment duration. The studied problem takes into account the uncertainty of the patients arrival time. A mixed integer linear program was proposed in addition to a Tabu Search (TS) meta-heuristic to solve the problem, for both cases deterministic and stochastic. Computational results on real world data for the deterministic case showed the efficiency of the proposed procedure.

In [35], authors investigated the physician rescheduling problem under the unplanned absences constraints (illness for example). The objective is to make the least possible adjustments on the initial schedule. The problem is formulated as mixed linear programming. This model is assessed over real world data, and the obtained results are near optimal solutions within a moderate computational time.

\section{Problem Definition}

In this section, the ICU department subject to this study is introduced. In addition, the current manual way used in generating the physician schedule is briefly described, as well as its shortcomings. Finally, the physician scheduling problem as it was presented by the ICU manager is introduced.

\subsection{The ICU Department}

The ICU department where the current study took place belongs to a local hospital. This ICU is composed of three separate buildings. These buildings are providing different services that are stated as follows:

1. Building 1: The main building of the ICU department.

2. Building 2: Severe cases of burned patients and women from difficult births are treated in this building.

3. Building 3: Specialized in treatment of sever cases of car accidents. In this building, a team of specialized physicians are ready to intervene outside of the ICU department.

The physicians load in the ICU department depends on the building type. Indeed, building 1 is the most busy one and requested special attention while preparing the physician schedule. In contrast, building 2 is less busy than building 1 in terms of load. The least busy building is building 3 . The detailed characteristics of the above-mentioned buildings as well as their impact on the physician schedule are presented in the "Problem mathematical formulation" subsection. 


\subsection{Manual Procedure}

The preparation of the physician scheduling is performed by a team led by an expert among the working staff (scheduler). The physician schedule preparation is a manual and a monthly cyclic procedure. This is because each month new highly qualified physicians are assigned to the ICU by the hospital mangers. The number of the assigned physicians is variable and depends on the needed physicians in the hospital containing the ICU. The variability in the number of assigned physicians imposes the first serious difficulty in the preparation of the physician schedule.

This physician schedule preparation procedure is a time consuming and a complicated task. Indeed, this process lasts more than two weeks of full working days. In addition, the required number of physicians for each building depends on the season (fall, winter, spring, and summer) and historical data are utilized to decide about these numbers. Furthermore, the hospital regulations, the healthcare services demand, and the preferences of the physicians generate hard and non-classical constraints. Therefore, meeting all of these constraints is a hard task.

The preferences of the physicians are communicated to the scheduler who is in charge of the scheduling procedure one month before the beginning of the schedule preparation. These preferences include days-off and vacations, desired shifts or sequences of shifts, and number of weekly hours. Consequently, a lot of contradictions are detected for the first iteration, and the schedule should be revised and corrected several times until reaching a schedule satisfying almost all the requirements and preferences.

Furthermore, the obtained schedules are usually unbalanced in term of workload, exhibiting an excessive overtime with all the financial consequences, and unsatisfied physicians since their preferences are not completely satisfied.

The consumed time in the manual preparation of the physician schedule and the procedure's shortcomings are the main motivations for the ICU department to switch to a new automated approach while generating the physician schedule. In addition, reducing the violation of the physicians preferences is particularly important, since physicians' satisfaction have an important impact on the delivered healthcare services quality. Moreover, a fair distribution of load between the physicians is highly desired to reduce the overtime and subsequently the associated cost.

\subsection{Problem Description}

The working hours in the studied ICU department are distributed into two shifts. These shifts are denoted $S_{1}$ and $S_{2}$, respectively. $S_{1}$ is a day shift starting from 7:00 a.m. and ending at 19:00 p.m. While $S_{2}$ is a night from 19:00 p.m. to 7:00 a.m.. In addition, the physicians ensure non-stopping services $24 \mathrm{~h}$ a day, 7 days a week.

At the beginning of each month, a set $P=\left\{P_{1}, P_{2}, \ldots P_{N}\right\}$ of $N$ physicians are assigned to the ICU department. The existing physicians are portioned by the scheduler into a set $T=\left\{T_{1}, T_{2}, T_{3}, T_{4}, T_{5}, T_{6}\right\}$ of six different teams. The parameter six teams is retrieved from historical data. The assigned physicians to the same team, should work together from the beginning until the end of month. This is a hard and non-classical constraint that limits the flexibility of the scheduling procedure.

The planning horizon is four weeks (one month) which is composed of a set $D=\left\{d_{1}, d_{2}, \ldots, d_{28}\right\}$ of 28 working days. For each shift $S_{j}(j=1,2)$, each day $d_{l}(l=1,2, \ldots, 28)$, each building $B_{k}(k=1,2,3)$, the scheduler should assign a unique team $T_{m}(m \in\{1,2, \ldots, 6\})$. The number of required physicians at building $B_{k}(k=1,2,3)$ during the shift $S_{j}$ is denoted $R_{k j}$.

Based on the accumulated experience and historical data, The ICU manager and the physician schedule preparation team desire to include the following constraints. It is worth noting that the majority of the following desired constraints are not satisfied in the manual schedule. These constraints are presented as follows. 
$\left(C_{1}\right)$ According to ICU regulations, the workload of each physician $P_{i}(i=1,2, \ldots, N)$ is $208 \mathrm{~h}$ per month.

$\left(C_{2}\right)$ Each physician $P_{i}(i=1,2, \ldots, N)$ is assigned to exactly one unique team during the planning horizon. This is to create a certain level of coordination and understanding between the members of each team. In other terms, the members of each team are unchanged for the horizon planning period (28 days).

$\left(C_{3}\right)$ Based on historical data, the number of physicians in each team is no less than 3 and no more than 6 . This is to ensure a minimum level of services in each building (no less than 3 ), and on the other hand, to not exceed the hospital capacity (no more than 6).

$\left(C_{4}\right)$ The assigned physicians to building $B_{2}$ and $B_{3}$ have to work only one shift per day including the weekends.

$\left(C_{5}\right)$ Only one shift per day is assigned for each physician $P_{i}(i=1,2, \ldots, N)$ at building $B_{1}$ during the weekdays: from Monday to Friday. This is due to the intensive workload in this building during the weekdays.

$\left(C_{6}\right)$ During the night shift $S_{2}$, only one team is assigned to serve the three buildings $B_{1}, B_{2}$, and $B_{3}$. This is because of the services demand drop during night shift.

$\left(C_{7}\right)$ The most sever cases are treated in building $B_{1}$. In order to ease the situation for the inpatients and to comfort them, the assigned team for $B_{1}$ at the beginning of the week has to stay there during all weekdays (from Monday to Friday).

$\left(C_{8}\right)$ A physician $P_{i}(i=1,2, \ldots, N)$ can not be assigned to building $B_{1}$ for two consecutive weeks. This is due to the intensive workload in this building.

$\left(C_{9}\right)$ Day and night shifts $S_{1}$ and $S_{2}$, on Saturday or Sunday, in building $B_{1}$, should be ensured by the same team. This team works $24 \mathrm{~h}$ during the assigned day (Saturday or Sunday). This heavy burden is taken into account throughout the two next preferences.

$\left(C_{10}\right)$ Each physician $P_{i}(i=1,2, \ldots, N)$ has two consecutive days off per week.

$\left(C_{11}\right)$ The team working on Saturday or Sunday at building $B_{1}$ should have a day-off before and day-off after. Such a team has to work only one day either Saturday or Sunday and not the entire weekend.

$\left(C_{12}\right)$ If a team works in buildings $B_{2}$ or $B_{3}$ Saturday during a shift, it should be assigned to $B_{2}$ or $B_{3}$ Sunday during a shift (the services demand in buildings $B_{2}$ and $B_{3}$ is less intense than in $B_{1}$ ).

$\left(C_{13}\right)$ If the night shift $S_{2}$ of a day $d_{l}(l \in\{1,2, \ldots, 28\})$ is covered by a team $T_{i}(i \in\{1,2, \ldots, 6\})$. In other terms, a team can not be assigned to two consecutive day and night shifts. This is to ensure the comfort of physicians.

$\left(C_{14}\right)$ During a day shift $S_{1}$, each physician $P_{i}(i=1,2, \ldots, N)$ is assigned at most to only one unique building (The workload for day shift is more important than night shift).

$\left(C_{15}\right)$ If a team is assigned to a building $B_{i}(i \in\{1,2,3\})$, for a day $d_{l}(l \in\{1,2, \ldots, 28\})$, and during a shift $S_{i}(i \in\{1,2\})$, then all the members of this team should have the same assignment.

$\left(C_{16}\right)$ A building $B_{i}(i \in\{1,2,3\})$ is served by a unique team at the same time (day and night shifts).

It is worth noting that during the preparation of the physician schedule, the assignment of teams to days, shifts, and building is simultaneous with the allocation of physicians to teams. Consequently, less flexibility and more difficulty are encountered while treating the studied problem compared to other classical personnel scheduling problems.

\section{Mathematical Modeling}

In this section, the definitions and the notations used in the rest of this paper are presented. In addition, mathematical formulation of the already defined problem is proposed. This mathematical formulation is an integer (binary) linear program one. 


\subsection{Definitions and Notations}

The following notations and definitions are adopted throughout the remaining of this paper.

- $\quad N$ is the number of physicians assigned to the ICU department at the month beginning.

- $\quad P=\left\{P_{1}, P_{2}, \ldots P_{N}\right\}$ is the set of physicians.

- $T=\left\{T_{1}, T_{2}, T_{3}, T_{4}, T_{5}, T_{6}\right\}$ is the set of teams.

- $D=\left\{d_{1}, d_{2}, \ldots, d_{28}\right\}$ is the set of days.

- $W=\left\{d_{k}: k=6+7 s, 7+7 s, s=0, \ldots, 3\right\}$ is the set of weekends (Saturday and Sunday of each week).

- $S=\left\{S_{1}, S_{2}\right\}$ is the set of shifts.

- $B=\left\{B_{1}, B_{2}, B_{3}\right\}$ is the set of buildings in the ICU department.

Seeking simplicity while expressing the constraints, the following notations will be adopted. For example, by $i \in P$, we mean physician $P_{i}$ with $i=1,2, \ldots, N$. The same way is extended to all the previous introduced sets: $T, D, W, S$, and $B$.

\subsection{Mathematical Model}

In this subsection, the physician scheduling problem is modeled as a binary linear program. To that aim, first the decision variables are defined. Finding the decision variables is the hardest part since these variables are reflecting the constraints and the way they are expressed. The number and the nature of these decision variables (positive, integer, binary, or a mix) impact enormously the quality of the obtained solution (optimal or near optimal or a feasible solution), and the consumed time while solving the studied problem. The selected decision variables are as follows.

- $Y_{i j k l m}=1$ if physician $P_{i}$ is assigned to shift $S_{j}$ in building $B_{k}$ at day $d_{l}$ in the team $T_{m}$, and 0 otherwise.

- $\quad Z_{i m}=1$ if physician $P_{i}$ is assigned to team $T_{m}$, and 0 otherwise.

- $V_{i l}=1$ if physician $P_{i}$ has two consecutive days off (within a week) starting from day $d_{l}$, and 0 otherwise.

- $\quad W_{i h m}=1$ if physicians $P_{i}$ and $P_{h}$ are assigned to the same team $T_{m}$, and 0 otherwise.

The decision variables $Y_{i j k l m}$ and $Z_{i m}$ are those indicating the assignment of the physicians to the days, shifts, buildings, and teams. However, the decision variables $V_{i l}$ are included to allow taking into account some physicians' preferences, as in taking two consecutive days off per week. The last decision variables $W_{i h m}$ are introduced to guarantee the same assignments (day, shift, building) for all physicians of the same team.

All the already mentioned constraints in section "Problem definition" and others, are expressed and presented in the sequel. It is worth noting that the latter mentioned constraints are those desired by the ICU manger and the physician scheduling team. The majority of these constraints are not satisfied while preparing the manual schedules. This is because of the faced hardness when including non-classical constraints. In addition, manually mixing all these complex constraints is hard and impossible to be reached in the majority of the treated cases.

1. Each physician $P_{i}(i=1,2, \ldots, N)$ is assigned to exactly one team:

$$
\sum_{m=1}^{6} Z_{i m}=1, \forall i \in P
$$

2. The number of teams' members is ranging from three to six depending on the building for which the team will be assigned.

$$
3 \leq \sum_{i=1}^{N} Z_{i m} \leq 6, \forall m \in T
$$


3. The decision variables $Y_{i j k l m}$ and $Z_{i m}$ are related by the following constraints:

$$
Y_{i j k l m} \leq Z_{i m}, \forall i \in P, j \in S, k \in B, l \in D, m \in T .
$$

The inequalities (3) are satisfied for all $i, j, k, l$, and $m$. Indeed, in the case where $Z_{\text {im }}=0$, then physician $P_{i}$ is not assigned to team $T_{m}$ and consequently $Y_{i j k l m}=0$ which is satisfied. On the other hand, there exists a unique team $m$ that should contain physician $P_{i}$ and some shifts, days, building such that $Y_{i j k l m}=1$ (otherwise $P_{i}$ has no work to do). This means that $Z_{i m}=1$. Consequently, the inequalities (3) are satisfied.

4. Each physician $P_{i}(i=1,2, \ldots, N)$ assigned to building $B_{2}$ or building $B_{3}$, works for exactly one shift per day.

$$
\sum_{j=1}^{2} Y_{i j k l m} \leq 1, \forall i \in P, \forall k=2,3, l \in D, m \in T .
$$

5. If a physician $P_{i}(i=1,2, \ldots, N)$ is assigned to building $B_{1}$ on weekdays (from Monday to Friday), then he works exactly one shift per day.

$$
\sum_{j=1}^{2} Y_{i j 1 l m} \leq 1 \forall i \in P, l \in D / W, m \in T \text {. }
$$

In the previous equation, $D / W$ denotes the set of days excluding the weekends.

6. All the buildings $B_{k}(k=1,2,3)$ are served by only one team for the night shifts.

$$
Y_{i 21 l m}=Y_{i 22 l m}=Y_{i 23 l m} \forall i \in P, l \in D, m \in T \text {. }
$$

7. According to the ICU regulations, each physician $P_{i}(i=1,2, \ldots, N)$ is requested to work at least for $208 \mathrm{~h}$ per month.

$$
\begin{gathered}
\sum_{j=1}^{2} \sum_{k=1}^{3} \sum_{l=1}^{28} \sum_{m=1}^{6} 12 Y_{i j k l m}- \\
\sum_{l=1}^{28} \sum_{m=1}^{6}\left(12 Y_{i 22 l m}+12 Y_{i 23 l m}\right) \geq 208,
\end{gathered}
$$

It is worth recalling that each shift's duration is $12 \mathrm{~h}$. In addition, constraints (6) are expressing the total workload for physician $P_{i}$, which takes into account constraints (6), by subtracting the expression $\sum_{l=1}^{28} \sum_{m=1}^{6}\left(12 Y_{i 22 l m}+12 Y_{i 23 l m}\right)$. Indeed, the night shift $S_{2}$ is covered by the same team in all buildings.

8. If a physician $P_{i}(i=1,2, \ldots, N)$ is assigned to a day shift in building $B_{1}$, he should ensure that shift day during all the weekdays (from Monday to Friday). This is for the comfort of the inpatients. Indeed, building $B_{1}$ is reserved to the treatment of the most sever cases. Consequently, dealing with the same physicians during the five weekdays is for the benefit of the inpatients.

$$
\begin{gathered}
Y_{i 11(1+7 s) m}=Y_{i 11(l+7 s) m}, \forall i \in P, \\
0 \leq s \leq 3,2 \leq l \leq 5, m \in T .
\end{gathered}
$$

In the previous constraints, $s$ is the week number, and $l$ is the day. For example, $s=0$ is the first week (in a month) and $l=3$ is day number 3 (Tuesday).

9. As a result of the intensity of the work and its complexity in building $B_{1}$, a physician $P_{i}(i=1,2, \ldots, N)$ should not be assigned there for two consecutive weeks.

$$
\begin{gathered}
Y_{i 11(1+7 s) m}+Y_{i 11(l+7(s+1)) l m} \leq 1, \forall i \in P, \\
0 \leq s \leq 3,1 \leq l \leq 5, m \in T .
\end{gathered}
$$


10. The required number of physicians in building $B_{k}$ during shift $S_{j}$ is $R_{k j}(k=1,2,3$ and $j=1,2)$.

$$
\sum_{i=1}^{N} Y_{i j k l m} \geq R_{k j}, \forall k \in B, l \in D, m \in T, j \in 1,2 .
$$

11. Each week, a physician $P_{i}(i=1,2, \ldots, N)$ has two consecutive days off.

$$
\sum_{l=1+7 s}^{(6+7 s)} V_{i l} \geq 1, \forall i \in P \text { and } 0 \leq s \leq 3
$$

In the latter constraints, $s$ refers to the week's index ( $s=0$ is the first week, $s=3$ is the fourth and last month's week).

12. The relationship between the decision variables $Y_{i j k l m}$ and $V_{i l}$ is expressed over the following constraints.

$$
\begin{gathered}
Y_{i 1 k l m}+Y_{i 2 s l m}+Y_{i 1 t(l+1) m}+Y_{i 2 w(l+1) m} \leq 4\left(1-V_{i l}\right), \\
\forall i \in P, l \in D /\{7,14,21,28\}, m \in T, k \in B, \\
t \in B, s \in B, w \in B .
\end{gathered}
$$

Indeed, if a physician $P_{i}(i=1,2, \ldots, N)$ is granted two consecutive days off, starting from day $d_{l}$ then $V_{i l}=1$. In this case, all the variables $Y_{i 1 k l m}, Y_{i 2(s) l m}, Y_{i 1 t(l+1) m}, Y_{i 2 w(l+1) m}$ are set to zero because in these two days $d_{l}$ and $d_{l+1}$ physician $P_{i}$ is not working.

13. During the weekends, the physician $P_{i}$ assigned to building $B_{1}$ for the day shift $S_{1}$ is also assigned to the night shift $S_{2}$.

$$
Y_{i 11 l m}=Y_{i 21 l m}, \forall i \in P, l \in W, m \in T .
$$

Recall that $W$ is the set of weekends.

14. As a result of the hardness of work in building $B_{1}$, a physician $P_{i}$ working there during Saturday or Sunday (weekend) should have a day-off before and a day-off after.

$$
\begin{aligned}
& Y_{i j k(l-1) m} \leq 1-Y_{i 11 l m}, \forall i \in P, \\
& j \in S, k \in B, l \in W, m \in T . \\
& Y_{i j k(l+1) m} \leq 1-Y_{i 11 l m}, \forall i \in P, \\
& j \in S, k \in B, l \in W /\{28\}, m \in T .
\end{aligned}
$$

15. A physician $P_{i}$ assigned to building $B_{2}$ or building $B_{3}$, working on Saturday during a day shift $S_{1}$, should work in the same building, during the day shift on Sunday.

$$
\begin{aligned}
Y_{i 1 k l m} & =Y_{i 1 k(l+1) m}, \forall i \in P, \\
k & \in B, l=\{6,13,20,27\}, m \in T .
\end{aligned}
$$

16. A physician $P_{i}$ assigned to a night shift $S_{2}$ during day $d_{l}$, should not work the day after $d_{(l+1)}$ during the day shift $S_{1}$. This is to ease the situation for physicians.

$$
\begin{gathered}
Y_{i 2 k l m}+Y_{i 1 s(l+l) m} \leq 1, \forall i \in P, \\
s, k \in B, l \in D /\{28\}, m \in T .
\end{gathered}
$$


17. During the day shift, a physician $P_{i}$ is assigned at most to only one unique building.

$$
Y_{i 11 l m}+Y_{i 12 l m}+Y_{i 13 l m} \leq 1, \forall i \in P, l \in D, m \in T .
$$

18. In order to ensure the same assignment (shift, day, building) of all members of the same team, the decision variables $W_{i h m}(i=1, \ldots, N, \forall i<h \leq N)$ are introduced. Recall that $W_{i h m}=1$ if physicians $P_{i}$ and $P_{h}$ are members of the same team $T_{m}$.

The first trivial resulting constraints mean that a couple of physicians may belong to the same team. Their corresponding expressions are as follows.

$$
\sum_{m=1}^{6} W_{i h m} \leq 1, \forall i \in P, \text { and } 1 \leq i<h \leq N .
$$

Since $W_{i h m}=W_{h i m}$, the condition $1 \leq i<h \leq N$ is introduced to avoid the repetition of the same couple twice.

The second type of constraints express a relationship between the decision variables $W_{i h m}$ and $Z_{i m}$. The corresponding expressions are presented as follows.

$$
Z_{i m}+Z_{h m} \leq W_{i h m}+1, \forall i \in P, i<h \leq N, m \in T .
$$

The latter constraints are satisfied. Indeed, if physicians $P_{i}$ and $P_{h}$ belong to the same team $T_{m}$, then $Z_{i m}=Z_{h m}=W_{i h m}=1$. Otherwise, physicians $P_{i}$ and $P_{h}$ do not belong to the same team. This means that $W_{i h m}=0$ for each team $T_{m}$, and $Z_{i m}+Z_{h m} \leq 1=W_{i h m}+1$.

The third type of constraints involve the same assignments (shift $S_{j}$, building $B_{k}$, and day $d_{l}$ ) for physicians belonging to the same team.

$$
\begin{gathered}
Y_{i j k l m}-Y_{h j k l m} \leq 1-W_{i h m}, \forall i \in P, \\
1 \leq i<h \leq N, j \in S, k \in B, l \in D, m \in T .
\end{gathered}
$$

In the case where physicians $P_{i}$ and $P_{h}$ do not belong to the same team $T_{m}$, then $W_{i h m}=0$. Consequently, $Y_{i j k l m}=Y_{h j k l m}=0$ and the latter constraints are satisfied. If $P_{i}$ and $P_{h}$ belong to the same team $T_{m}$ then $W_{i h m}=1$. In this case, physicians $P_{i}$ and $P_{h}$ have the same assignment and $Y_{i j k l m}=Y_{h j k l m}=0$.

The following constraints hold.

$$
\begin{aligned}
& W_{i h m} \leq Z_{i m}, \forall i \in P, 1 \leq i<h \leq N, m \in T \\
& W_{i h m} \leq Z_{h m}, \forall i \in P, 1 \leq i<h \leq N, m \in T
\end{aligned}
$$

The objective to be minimized is the total overtime which is expressed as follows.

$$
\begin{gathered}
Z=\sum_{i=1}^{N} \sum_{j=1}^{2} \sum_{k=1}^{3} \sum_{l=1}^{28} \sum_{m=1}^{6} 12 Y_{i j k l m}- \\
\sum_{i=1 l=1}^{N} \sum_{m=1}^{28}\left(12 Y_{i 22 l m}+12 Y_{i 23 l m}\right)-208 N
\end{gathered}
$$

In the expression of the objective function (23), $208 \mathrm{~N}$ represents the minimum workload of $N$ physicians (7).

In addition, during the night shift, the same team is covering all the buildings (6), thus the expression: $\sum_{i=1}^{N} \sum_{l=1}^{28} \sum_{m=1}^{6}\left(12 Y_{i 22 l m}+12 Y_{i 23 l m}\right)$ should be subtracted from: $\sum_{i=1}^{N} \sum_{j=1}^{2} \sum_{k=1}^{3} \sum_{l=1}^{28} \sum_{m=1}^{6} 12 Y_{i j k l m}$. Therefore, $Z$ represents the total overtime load. 
The presented mathematical model (1-23) might be generalized in terms of performances, constraints, and preferences. Indeed, the overtime objective function could be replaced by any other objective function as the total unbalanced load, a multi-objective function combining overtime and other performance measures as the physicians' satisfaction function (how much the preferences are satisfied) with different weights. The existing set of constraints could be extended to include other new constraints; for example, the total allocated personnel budget for the ICU department. The physicians' preferences are subject to generalization, for example allocating short period off to attend local or international seminars or training is among these preferences. It is worth noting that the proposed mathematical model is representing an NP-Hard combinatorial optimization problem. Thus, the generalization of such a model might increase its complexity and therefore its resolution. Therefore, these generalizations should be carefully studied to avoid such a problem.

\section{Computational Results and Interpretation}

In this section, the efficiency and the performance of the proposed mathematical model is assessed over experimental tests. These experimental tests are carried out on real world data, provided by the ICU department, This data is presented in the following subsection.

\subsection{Test Problems}

The required data are provided by the ICU department (ICU physician scheduling team). These data are composed of months and the corresponding number of assigned physicians to ICU. In addition, the manual physician schedules for each month are also provided. The most important months are those with limited physician number (scarce of physicians in the hospital compared to the required services demand). These months, and due to limited resources, are considered as a test of the efficiency of any proposed physician scheduling procedure. In this case study, the data related to September and October are provided. Indeed, September is the month with the lowest number of assigned physicians to the ICU.

Providing data for only two months may appear insufficient and therefore accurate and useful conclusions could not be reached. In this context, recall that the most important resource parameter in this study is the number of allocated physicians $(N)$ to the ICU department at the beginning of each month. The month of September is presenting the lowest number with $N=18$ and dealing with it is of capital interest. This is because the obtained schedule will provide a precise idea about the utilization of the least number of provided physicians (scarce resource).

The number of assigned physicians to ICU during September is $N=18$, this is the minimum required number. Indeed, 6 teams should be formed and at least 3 physicians are assigned to each team. For October, $N=24$ physicians are assigned to ICU and this is considered among the largest assigned number. The manual physician schedules corresponding to September and October are displayed in Tables 1 and 2, respectively. In tables (Tables 1 and 2), a team $T_{m}$ is assigned to each combination of a day $d_{l}(l=1,2, \ldots, 28)$, a building $B_{k}(k=1,2,3)$, and a shift $S_{j}(j=1,2)$.

The integer linear program model is coded with LINGO software, and implemented on a personnel computer with 1GB RAM and a processor Pentium IV $2.8 \mathrm{GHz}$.

At this stage, it is worth mentioning that the presented constraints $C_{1}, C_{2}, \ldots, C_{16}$ in subsection "Problem description" are almost not satisfied in the proposed manual schedules. This is due to the complexity of the required constraints and their manual implementation.

In this context, and according to Table 1 , we observe that the physicians of team $T_{6}$ have taken only five days off out of eight, during the whole month. These days off are: $d_{7}, d_{20}, d_{22}, d_{23}$, and $d_{26}$. In addition, the only two consecutive days off are $d_{22}, d_{23}$ for $T_{6}$. Thus, constraints (11) are not satisfied.

Furthermore, physicians of team $T_{1}$ are assigned to the night shift $S_{2}$ of day $d_{1}$ and also to day shift $S_{1}$ on day $d_{2}$, which is not satisfying the constraints (17). Moreover, team $T_{2}$ is only assigned to 12 shifts with a total workload $144 \mathrm{~h}$ during September which is far below the requested workload. 
These two observations are cited to highlight the negative consequences of the manual schedules, especially in satisfying the physicians' preferences. A more detailed analysis will be proposed in the "Results and analysis" subsection.

Table 1. Manual physician schedule for September.

\begin{tabular}{|c|c|c|c|c|c|c|}
\hline & \multicolumn{2}{|c|}{$B_{1}$} & \multicolumn{2}{|c|}{$B_{2}$} & \multicolumn{2}{|c|}{$B_{3}$} \\
\hline Day & $S_{1}$ & $S_{2}$ & $S_{1}$ & $S_{2}$ & $S_{1}$ & $S_{2}$ \\
\hline 1 & $T_{6}$ & $T_{1}$ & $T_{3}$ & $T_{1}$ & $T_{4}$ & $T_{1}$ \\
\hline 2 & $T_{6}$ & $T_{4}$ & $T_{2}$ & $T_{4}$ & $T_{1}$ & $T_{4}$ \\
\hline 3 & $T_{6}$ & $T_{5}$ & $T_{2}$ & $T_{5}$ & $T_{1}$ & $T_{5}$ \\
\hline 4 & $T_{6}$ & $T_{5}$ & $T_{2}$ & $T_{5}$ & $T_{1}$ & $T_{5}$ \\
\hline 5 & $T_{6}$ & $T_{4}$ & $T_{2}$ & $T_{4}$ & $T_{1}$ & $T_{4}$ \\
\hline 6 & $T_{6}$ & $T_{6}$ & $T_{5}$ & $T_{6}$ & $T_{1}$ & $T_{6}$ \\
\hline 7 & $T_{2}$ & $T_{2}$ & $T_{5}$ & $T_{2}$ & $T_{1}$ & $T_{2}$ \\
\hline 8 & $T_{3}$ & $T_{5}$ & $T_{1}$ & $T_{5}$ & $T_{6}$ & $T_{5}$ \\
\hline 9 & $T_{3}$ & $T_{5}$ & $T_{1}$ & $T_{5}$ & $T_{6}$ & $T_{5}$ \\
\hline 10 & $T_{3}$ & $T_{2}$ & $T_{1}$ & $T_{2}$ & $T_{6}$ & $T_{2}$ \\
\hline 11 & $T_{3}$ & $T_{2}$ & $T_{1}$ & $T_{2}$ & $T_{6}$ & $T_{2}$ \\
\hline 12 & $T_{3}$ & $T_{5}$ & $T_{1}$ & $T_{5}$ & $T_{6}$ & $T_{5}$ \\
\hline 13 & $T_{3}$ & $T_{3}$ & $T_{2}$ & $T_{3}$ & $T_{6}$ & $T_{3}$ \\
\hline 14 & $T_{1}$ & $T_{1}$ & $T_{2}$ & $T_{1}$ & $T_{6}$ & $T_{1}$ \\
\hline 15 & $T_{4}$ & $T_{2}$ & $T_{6}$ & $T_{2}$ & $T_{3}$ & $T_{2}$ \\
\hline 16 & $T_{4}$ & $T_{2}$ & $T_{6}$ & $T_{2}$ & $T_{3}$ & $T_{2}$ \\
\hline 17 & $T_{4}$ & $T_{1}$ & $T_{6}$ & $T_{1}$ & $T_{3}$ & $T_{1}$ \\
\hline 18 & $T_{4}$ & $T_{1}$ & $T_{6}$ & $T_{1}$ & $T_{3}$ & $T_{1}$ \\
\hline 19 & $T_{4}$ & $T_{2}$ & $T_{6}$ & $T_{2}$ & $T_{3}$ & $T_{2}$ \\
\hline 20 & $T_{4}$ & $T_{4}$ & $T_{1}$ & $T_{4}$ & $T_{3}$ & $T_{4}$ \\
\hline 21 & $T_{6}$ & $T_{6}$ & $T_{1}$ & $T_{6}$ & $T_{3}$ & $T_{6}$ \\
\hline 22 & $T_{5}$ & $T_{1}$ & $T_{3}$ & $T_{1}$ & $T_{4}$ & $T_{1}$ \\
\hline 23 & $T_{5}$ & $T_{1}$ & $T_{3}$ & $T_{1}$ & $T_{4}$ & $T_{1}$ \\
\hline 24 & $T_{5}$ & $T_{6}$ & $T_{3}$ & $T_{6}$ & $T_{4}$ & $T_{6}$ \\
\hline 25 & $T_{5}$ & $T_{6}$ & $T_{3}$ & $T_{6}$ & $T_{4}$ & $T_{6}$ \\
\hline 26 & $T_{5}$ & $T_{1}$ & $T_{3}$ & $T_{1}$ & $T_{4}$ & $T_{1}$ \\
\hline 27 & $T_{5}$ & $T_{5}$ & $T_{6}$ & $T_{5}$ & $T_{4}$ & $T_{5}$ \\
\hline 28 & $T_{3}$ & $T_{3}$ & $T_{6}$ & $T_{3}$ & $T_{4}$ & $T_{3}$ \\
\hline
\end{tabular}

\subsection{Results and Analysis}

The optimized physician schedules for September and October are those obtained by implementation of the integer linear programming model. The obtained schedules are presented respectively in Tables 3 and 4.

The number of assigned physicians to ICU during September is the lowest one $(N=18)$ compared to other months. As a result of this reason, a particular attention will be paid to September and a related detailed analysis will be presented for this particular month.

The presented results in Tables 1 and 3 are analyzed and summarized respectively in Tables 5 and 6 . Tables 5 and 6 are reporting the summary for the manual and optimized procedures, respectively. 
This first analysis is performed while focusing on the overtime, under-load, and unbalanced workload. Recall that the overtime is the objective function to be minimized.

The parameters allowing the comparison between the manual and the optimized procedure are presented as follows.

- $\quad N S_{m}$ : The number of shifts performed by each physician in team $T_{m}(m=1, \ldots, 6)$.

- $\quad N P_{m}$ : The number of physicians in team $T_{m}(m=1, \ldots, 6)$.

- $O T_{m}$ : The overtime (hours) for each physician of team $T_{m}(m=1, \ldots, 6)$, and we have $O T_{m}=$ $\max \left(N S_{m} \times 12-208,0\right)$.

- $U L_{m}$ : the under-load of each physician of team $T_{m}(m=1, \ldots, 6)$, which is expressed as: $U L_{m}=$ $N S_{m} \times 12-208$ (with a negative sign).

Table 2. Manual physician schedule for October.

\begin{tabular}{|c|c|c|c|c|c|c|}
\hline & \multicolumn{2}{|c|}{$B_{1}$} & \multicolumn{2}{|c|}{$B_{2}$} & \multicolumn{2}{|c|}{$B_{3}$} \\
\hline Day & $S_{1}$ & $S_{2}$ & $S_{1}$ & $S_{2}$ & $S_{1}$ & $S_{2}$ \\
\hline 1 & $T_{2}$ & $T_{6}$ & $T_{4}$ & $T_{6}$ & $T_{5}$ & $T_{6}$ \\
\hline 2 & $T_{2}$ & $T_{6}$ & $T_{4}$ & $T_{6}$ & $T_{5}$ & $T_{6}$ \\
\hline 3 & $T_{2}$ & $T_{3}$ & $T_{4}$ & $T_{3}$ & $T_{5}$ & $T_{3}$ \\
\hline 4 & $T_{2}$ & $T_{3}$ & $T_{4}$ & $T_{3}$ & $T_{5}$ & $T_{3}$ \\
\hline 5 & $T_{2}$ & $T_{6}$ & $T_{4}$ & $T_{6}$ & $T_{5}$ & $T_{6}$ \\
\hline 6 & $T_{2}$ & $T_{2}$ & $T_{3}$ & $T_{2}$ & $T_{5}$ & $T_{2}$ \\
\hline 7 & $T_{4}$ & $T_{4}$ & $T_{3}$ & $T_{4}$ & $T_{5}$ & $T_{4}$ \\
\hline 8 & $T_{1}$ & $T_{3}$ & $T_{5}$ & $T_{3}$ & $T_{2}$ & $T_{3}$ \\
\hline 9 & $T_{1}$ & $T_{3}$ & $T_{5}$ & $T_{3}$ & $T_{2}$ & $T_{3}$ \\
\hline 10 & $T_{1}$ & $T_{4}$ & $T_{5}$ & $T_{4}$ & $T_{2}$ & $T_{4}$ \\
\hline 11 & $T_{1}$ & $T_{4}$ & $T_{5}$ & $T_{4}$ & $T_{2}$ & $T_{4}$ \\
\hline 12 & $T_{1}$ & $T_{3}$ & $T_{5}$ & $T_{3}$ & $T_{2}$ & $T_{3}$ \\
\hline 13 & $T_{1}$ & $T_{1}$ & $T_{4}$ & $T_{1}$ & $T_{2}$ & $T_{1}$ \\
\hline 14 & $T_{5}$ & $T_{5}$ & $T_{4}$ & $T_{5}$ & $T_{2}$ & $T_{5}$ \\
\hline 15 & $T_{2}$ & $T_{6}$ & $T_{4}$ & $T_{6}$ & $T_{5}$ & $T_{6}$ \\
\hline 16 & $T_{2}$ & $T_{6}$ & $T_{4}$ & $T_{6}$ & $T_{5}$ & $T_{6}$ \\
\hline 17 & $T_{2}$ & $T_{3}$ & $T_{4}$ & $T_{3}$ & $T_{5}$ & $T_{3}$ \\
\hline 18 & $T_{2}$ & $T_{3}$ & $T_{4}$ & $T_{3}$ & $T_{5}$ & $T_{3}$ \\
\hline 19 & $T_{2}$ & $T_{6}$ & $T_{4}$ & $T_{6}$ & $T_{5}$ & $T_{6}$ \\
\hline 20 & $T_{2}$ & $T_{2}$ & $T_{3}$ & $T_{2}$ & $T_{5}$ & $T_{2}$ \\
\hline 21 & $T_{4}$ & $T_{4}$ & $T_{3}$ & $T_{4}$ & $T_{5}$ & $T_{4}$ \\
\hline 22 & $T_{1}$ & $T_{3}$ & $T_{5}$ & $T_{3}$ & $T_{2}$ & $T_{3}$ \\
\hline 23 & $T_{1}$ & $T_{3}$ & $T_{5}$ & $T_{3}$ & $T_{2}$ & $T_{3}$ \\
\hline 24 & $T_{1}$ & $T_{4}$ & $T_{5}$ & $T_{4}$ & $T_{2}$ & $T_{4}$ \\
\hline 25 & $T_{1}$ & $T_{4}$ & $T_{5}$ & $T_{4}$ & $T_{2}$ & $T_{4}$ \\
\hline 26 & $T_{1}$ & $T_{3}$ & $T_{5}$ & $T_{3}$ & $T_{2}$ & $T_{3}$ \\
\hline 27 & $T_{1}$ & $T_{1}$ & $T_{4}$ & $T_{1}$ & $T_{2}$ & $T_{1}$ \\
\hline 28 & $T_{5}$ & $T_{5}$ & $T_{4}$ & $T_{5}$ & $T_{2}$ & $T_{5}$ \\
\hline
\end{tabular}


Table 3. Optimized physician schedule for September.

\begin{tabular}{|c|c|c|c|c|c|c|}
\hline & \multicolumn{2}{|c|}{$B_{1}$} & \multicolumn{2}{|c|}{$B_{2}$} & \multicolumn{2}{|c|}{$B_{3}$} \\
\hline Day & $S_{1}$ & $S_{2}$ & $S_{1}$ & $S_{2}$ & $S_{1}$ & $S_{2}$ \\
\hline 1 & $T_{1}$ & $T_{4}$ & $T_{3}$ & $T_{4}$ & $T_{6}$ & $T_{4}$ \\
\hline 2 & $T_{1}$ & $T_{4}$ & $T_{6}$ & $T_{4}$ & $T_{3}$ & $T_{4}$ \\
\hline 3 & $T_{1}$ & $T_{5}$ & $T_{3}$ & $T_{5}$ & $T_{6}$ & $T_{5}$ \\
\hline 4 & $T_{1}$ & $T_{3}$ & $T_{2}$ & $T_{3}$ & $T_{4}$ & $T_{3}$ \\
\hline 5 & $T_{1}$ & $T_{3}$ & $T_{5}$ & $T_{3}$ & $T_{2}$ & $T_{3}$ \\
\hline 6 & $T_{6}$ & $T_{6}$ & $T_{2}$ & $T_{6}$ & $T_{5}$ & $T_{6}$ \\
\hline 7 & $T_{4}$ & $T_{4}$ & $T_{2}$ & $T_{4}$ & $T_{5}$ & $T_{4}$ \\
\hline 8 & $T_{3}$ & $T_{6}$ & $T_{2}$ & $T_{6}$ & $T_{5}$ & $T_{6}$ \\
\hline 9 & $T_{3}$ & $T_{4}$ & $T_{2}$ & $\mathrm{~T}_{4}$ & $T_{5}$ & $T_{4}$ \\
\hline 10 & $T_{3}$ & $T_{2}$ & $T_{5}$ & $T_{2}$ & $T_{1}$ & $T_{2}$ \\
\hline 11 & $T_{3}$ & $T_{6}$ & $T_{5}$ & $T_{6}$ & $T_{1}$ & $T_{6}$ \\
\hline 12 & $T_{3}$ & $T_{5}$ & $T_{4}$ & $T_{5}$ & $T_{1}$ & $T_{5}$ \\
\hline 13 & $T_{2}$ & $T_{2}$ & $T_{6}$ & $T_{2}$ & $T_{4}$ & $T_{2}$ \\
\hline 14 & $T_{1}$ & $T_{1}$ & $T_{6}$ & $T_{1}$ & $T_{4}$ & $T_{1}$ \\
\hline 15 & $T_{6}$ & $T_{5}$ & $T_{4}$ & $T_{5}$ & $T_{2}$ & $T_{5}$ \\
\hline 16 & $T_{6}$ & $T_{5}$ & $T_{2}$ & $T_{5}$ & $T_{3}$ & $T_{5}$ \\
\hline 17 & $T_{6}$ & $T_{2}$ & $T_{4}$ & $T_{2}$ & $T_{1}$ & $T_{2}$ \\
\hline 18 & $T_{6}$ & $T_{5}$ & $T_{4}$ & $T_{5}$ & $T_{1}$ & $T_{5}$ \\
\hline 19 & $T_{6}$ & $T_{5}$ & $T_{3}$ & $T_{5}$ & $T_{1}$ & $T_{5}$ \\
\hline 20 & $T_{2}$ & $T_{2}$ & $T_{1}$ & $T_{2}$ & $T_{3}$ & $T_{2}$ \\
\hline 21 & $T_{4}$ & $T_{4}$ & $T_{1}$ & $T_{4}$ & $T_{3}$ & $T_{4}$ \\
\hline 22 & $T_{2}$ & $T_{5}$ & $T_{1}$ & $T_{5}$ & $T_{6}$ & $T_{5}$ \\
\hline 23 & $T_{2}$ & $T_{1}$ & $T_{6}$ & $T_{1}$ & $T_{3}$ & $T_{1}$ \\
\hline 24 & $T_{2}$ & $T_{6}$ & $T_{5}$ & $T_{6}$ & $T_{4}$ & $T_{6}$ \\
\hline 25 & $T_{2}$ & $T_{6}$ & $T_{5}$ & $T_{6}$ & $T_{4}$ & $T_{6}$ \\
\hline 26 & $T_{2}$ & $T_{6}$ & $T_{3}$ & $T_{6}$ & $T_{4}$ & $T_{6}$ \\
\hline 27 & $T_{1}$ & $T_{1}$ & $T_{3}$ & $T_{1}$ & $T_{4}$ & $T_{1}$ \\
\hline 28 & $T_{5}$ & $T_{5}$ & $T_{3}$ & $T_{5}$ & $T_{4}$ & $T_{5}$ \\
\hline
\end{tabular}

Tables 5 and 6 show that the total overtime is reduced from $576 \mathrm{~h}$ to $288 \mathrm{~h}$, which is a reduction of $50 \%$. The under-load which was $\mathbf{- 2 8 8}$ is totally eliminated $(0 \mathrm{~h})$. In addition, the number of covered shifts by each physician is now ranging from 18 to 19 instead of ranging between 13 and 25 for the manual schedule. This provides a strong evidence that the workload is now balanced and almost fair within the physicians community.

A similar analysis of the obtained results for October shows that there is a reduction of the over time by about $69.23 \%$. Furthermore, the under-load which used to be $-648 \mathrm{~h}$ for the manual way, is now completely eliminated. The number of shifts which varies from 6 to 28 for the manual way, is now ranging between 18 and 19 shifts. Furthermore, the consumed time while preparing the physician schedule using the optimized procedure, is not exceeding two hours instead of more than two weeks for the manual procedure.

A more detailed analysis is focusing on other parameters, as the total days off, and the total night shifts for each physician. These parameters are for a high interest especially for the physicians. 
This analysis is based on Tables 7 and 8, which are corresponding to manual and optimized ways, respectively. The parameters appearing in these two tables (Tables 7 and 8 ) are defined as follows.

- $\quad N S_{m}$ : The number of shifts performed by each physician in team $T_{m}(m=1, \ldots, 6)$.

- $\quad N N S_{m}$ : The number of night shifts covered by any physician in team $T_{m}(m=1, \ldots, 6)$.

- $\quad N D O_{m}$ : The number of days off for each physician in team $T_{m}(m=1, \ldots, 6)$.

- $\quad M D O_{m}$ : The maximum duration between two consecutive days off for each physician in team $T_{m}(m=1, \ldots, 6)$.

- $m D O_{m}$ : The minimum duration between two consecutive days off for each physician in team $T_{m}$ $(m=1, \ldots, 6)$.

Table 4. Optimized physician schedule for October.

\begin{tabular}{|c|c|c|c|c|c|c|}
\hline & \multicolumn{2}{|c|}{$B_{1}$} & \multicolumn{2}{|c|}{$B_{2}$} & \multicolumn{2}{|c|}{$B_{3}$} \\
\hline Day & $S_{1}$ & $S_{2}$ & $S_{1}$ & $S_{2}$ & $S_{1}$ & $S_{2}$ \\
\hline 1 & $T_{1}$ & $T_{4}$ & $T_{3}$ & $T_{4}$ & $T_{5}$ & $T_{4}$ \\
\hline 2 & $T_{1}$ & $T_{5}$ & $T_{3}$ & $T_{5}$ & $T_{2}$ & $T_{5}$ \\
\hline 3 & $T_{1}$ & $T_{3}$ & $T_{6}$ & $T_{3}$ & $T_{2}$ & $T_{3}$ \\
\hline 4 & $T_{1}$ & $T_{6}$ & $T_{2}$ & $T_{6}$ & $T_{4}$ & $T_{6}$ \\
\hline 5 & $T_{1}$ & $T_{2}$ & $T_{5}$ & $T_{2}$ & $T_{4}$ & $T_{2}$ \\
\hline 6 & $T_{6}$ & $T_{6}$ & $T_{4}$ & $T_{6}$ & $T_{5}$ & $T_{6}$ \\
\hline 7 & $T_{3}$ & $T_{3}$ & $T_{4}$ & $T_{3}$ & $T_{5}$ & $T_{3}$ \\
\hline 8 & $T_{6}$ & $T_{5}$ & $T_{1}$ & $T_{5}$ & $T_{4}$ & $T_{5}$ \\
\hline 9 & $T_{6}$ & $T_{5}$ & $T_{1}$ & $T_{5}$ & $T_{3}$ & $T_{5}$ \\
\hline 10 & $T_{6}$ & $T_{5}$ & $T_{3}$ & $T_{5}$ & $T_{2}$ & $T_{5}$ \\
\hline 11 & $T_{6}$ & $T_{1}$ & $T_{2}$ & $T_{1}$ & $T_{4}$ & $T_{1}$ \\
\hline 12 & $T_{6}$ & $T_{5}$ & $T_{2}$ & $T_{5}$ & $T_{4}$ & $T_{5}$ \\
\hline 13 & $T_{3}$ & $T_{3}$ & $T_{2}$ & $T_{3}$ & $T_{4}$ & $T_{3}$ \\
\hline 14 & $T_{1}$ & $T_{1}$ & $T_{2}$ & $T_{1}$ & $T_{4}$ & $T_{1}$ \\
\hline 15 & $T_{3}$ & $T_{4}$ & $T_{5}$ & $T_{4}$ & $T_{6}$ & $T_{4}$ \\
\hline 16 & $T_{3}$ & $T_{4}$ & $T_{5}$ & $T_{4}$ & $T_{6}$ & $T_{4}$ \\
\hline 17 & $T_{3}$ & $T_{1}$ & $T_{2}$ & $T_{1}$ & $T_{6}$ & $T_{1}$ \\
\hline 18 & $T_{3}$ & $T_{1}$ & $T_{2}$ & $T_{1}$ & $T_{6}$ & $T_{1}$ \\
\hline 19 & $T_{3}$ & $T_{6}$ & $T_{5}$ & $T_{6}$ & $T_{2}$ & $T_{6}$ \\
\hline 20 & $T_{1}$ & $T_{1}$ & $T_{2}$ & $T_{1}$ & $T_{5}$ & $T_{1}$ \\
\hline 21 & $T_{4}$ & $T_{4}$ & $T_{2}$ & $T_{4}$ & $T_{5}$ & $T_{4}$ \\
\hline 22 & $T_{5}$ & $T_{3}$ & $T_{2}$ & $T_{3}$ & $T_{1}$ & $T_{3}$ \\
\hline 23 & $T_{5}$ & $T_{2}$ & $T_{1}$ & $T_{2}$ & $T_{4}$ & $T_{2}$ \\
\hline 24 & $T_{5}$ & $T_{4}$ & $T_{1}$ & $T_{4}$ & $T_{3}$ & $T_{4}$ \\
\hline 25 & $T_{5}$ & $T_{1}$ & $T_{6}$ & $T_{1}$ & $T_{3}$ & $T_{1}$ \\
\hline 26 & $T_{5}$ & $T_{1}$ & $T_{6}$ & $T_{1}$ & $T_{2}$ & $T_{1}$ \\
\hline 27 & $T_{4}$ & $T_{4}$ & $T_{2}$ & $T_{4}$ & $T_{6}$ & $T_{4}$ \\
\hline 28 & $T_{3}$ & $T_{3}$ & $T_{2}$ & $T_{3}$ & $T_{6}$ & $T_{3}$ \\
\hline
\end{tabular}


Table 5. Analysis of the manual physician schedule for September.

\begin{tabular}{ccccc}
\hline Team & $\boldsymbol{N} S_{m}$ & $\boldsymbol{N} \boldsymbol{P}_{\boldsymbol{m}}$ & $\boldsymbol{O} \boldsymbol{T}_{\boldsymbol{m}}$ & $\boldsymbol{U} \boldsymbol{L}_{\boldsymbol{m}}$ \\
\hline$T_{1}$ & 21 & 3 & 132 & 0 \\
\hline$T_{2}$ & 13 & 3 & 0 & -156 \\
\hline$T_{3}$ & 22 & 3 & 168 & 0 \\
\hline$T_{4}$ & 17 & 3 & 0 & -12 \\
\hline$T_{5}$ & 14 & 3 & 0 & -120 \\
\hline$T_{6}$ & 25 & 3 & 276 & 0 \\
\hline & Total & & $\mathbf{5 7 6}$ & $\mathbf{- 2 8 8}$ \\
\hline
\end{tabular}

Table 6. Analysis of the optimized physician schedule for September.

\begin{tabular}{ccccc}
\hline Team & $\boldsymbol{N} \boldsymbol{S}_{\boldsymbol{m}}$ & $\boldsymbol{N} \boldsymbol{P}_{\boldsymbol{m}}$ & $\boldsymbol{O} \boldsymbol{T}_{\boldsymbol{m}}$ & $\boldsymbol{U} \boldsymbol{L}_{\boldsymbol{m}}$ \\
\hline$T_{1}$ & 19 & 3 & 20 & 0 \\
\hline$T_{2}$ & 19 & 3 & 20 & 0 \\
\hline$T_{3}$ & 18 & 3 & 8 & 0 \\
\hline$T_{4}$ & 19 & 3 & 20 & 0 \\
\hline$T_{5}$ & 18 & 3 & 8 & 0 \\
\hline$T_{6}$ & 19 & 3 & 20 & 0 \\
\hline & Total & & $\mathbf{2 8 8}$ & 0 \\
\hline
\end{tabular}

Table 7. Detailed analysis of the optimized physician schedule for September.

\begin{tabular}{cccccc}
\hline Team & $N S_{m}$ & $N N S_{m}$ & $N^{2} O_{m}$ & $\mathbf{M D O}_{m}$ & $\mathbf{m D O}_{m}$ \\
\hline$T_{1}$ & 19 & 3 & 11 & 7 & 1 \\
\hline$T_{2}$ & 19 & 4 & 11 & 7 & 1 \\
\hline$T_{3}$ & 18 & 2 & 10 & 5 & 1 \\
\hline$T_{4}$ & 19 & 5 & 11 & 4 & 1 \\
\hline$T_{5}$ & 18 & 8 & 11 & 8 & 1 \\
\hline$T_{6}$ & 19 & 6 & 10 & 7 & 1 \\
\hline
\end{tabular}

Based on Tables 7 and 8, one could observe that the number of days off is ranging from 5 to 16 days for the manual procedure, which is a wide range of variability. Instead of that, for the optimized schedule, the number of days off is varying from 10 to 11, which indicates the fairness of the new schedule. In addition, in some cases a team can spend more than 12 days of continuous work to get a day off. This is the case of $T_{1}$ which works without interruption from $d_{1}$ until $d_{12}$ to be granted a day off in $d_{13}$. In contrast, for the new way preparing the schedule, the same duration is reduced to 8 days.

The same analysis is carried out for October. Surprisingly, for the manual procedure, the number of days off is ranging from $2\left(T_{2}\right)$ to $22\left(T_{6}\right)$, which is unacceptable. For the optimized procedure, the number of days off are ranging from 9 to 12. Furthermore, the maximum duration between two consecutive vacations is reduced from 13 days to 8 days. 
Table 8. Detailed analysis of the manual physician schedule for September.

\begin{tabular}{cccccc}
\hline Team & $N S_{m}$ & $N N S_{m}$ & $N^{2} O_{m}$ & $M^{2} O_{m}$ & $m D O_{m}$ \\
\hline$T_{1}$ & 21 & 7 & 8 & 12 & 1 \\
\hline$T_{2}$ & 13 & 6 & 16 & 4 & 1 \\
\hline$T_{3}$ & 22 & 2 & 8 & 12 & 6 \\
\hline$T_{4}$ & 17 & 3 & 12 & 6 & 1 \\
\hline$T_{5}$ & 14 & 6 & 15 & 6 & 1 \\
\hline$T_{6}$ & 25 & 4 & 5 & 12 & 2 \\
\hline
\end{tabular}

The presented results on real world data provide strong evidence of dramatic enhancements observed by adopting the optimized procedure. These enhancements are concerning the cost since the total overtime is reduced. In addition, the workload is now balanced, and the physicians' preferences (week vacations) are taken into account effectively.

Furthermore, each one of the real-life studied personnel scheduling problems has its own characteristics. Therefore, these problems have a little common parts (decision variables, constraints, objective function). Consequently, the comparison of the obtained results for each problem with others is quite difficult. This is the case of the current studied problem.

\subsection{Adaptability and Flexibility of the Proposed Procedure}

In order to adapt the already presented procedure to responding efficiently and rapidly to unpredictable human and/or external factors, three scenarios and their corresponding adaptations are presented. The unpredictable scenarios are: (1) absence of a physician, (2) a medium scale disaster such as fire, flood, earthquake, and (3) a large scale event such as a pandemic. The main objective of this subsection is to provide a quick response to these unpredictable events. In this context, the already obtained optimal schedule is adjusted to cope with the new situation.

\subsubsection{Absence of a Physician}

In the case where a physician (for any reason) is declared absent for a limited period, the already obtained schedule is modified for each day of absence as follows.

1. A team not working this day is selected (there are two teams).

2. This team is the one with the minimum shifts (to reduce the incurred over time).

3. This team is not working the shift before (to avoid having two consecutive working shifts).

4. Select one of the physicians within this team.

One of the three last conditions may be dropped in order to have a feasible solution.

Example 1. At day $d_{8}$ of September, the manager learns that a physician from team $T_{3}$ is sick and will be absent for days 8-7-9. The manager action following the previous 4 steps is presented below.

1. The teams not working in day $d_{8}$ are $T_{4}$ and $T_{1}$.

2. The two teams have the same number of shifts (19 shifts).

3. The team which is not working the shift before is $T_{1}$.

4. Call one of the physicians within team $T_{1}$ to join team $T_{3}$ during the day shift at building 1 .

Following the same procedure, a physician from team $T_{1}$ is assigned to cover day $d_{9}$ with team $T_{3}$. During day $d_{10}$, a physician of team $T_{6}$ is charged to replace his absent colleague. 


\subsubsection{Medium Scale Disaster}

To overcome some unpredictable and dangerous events such as fire, flood, earthquake, the obtained optimal schedule could be adapted rapidly to respond to the dramatic increasing in service demand by providing more resources. This is achieved by adding a non-working teams for each shift as follows. Each one of the two non-working teams (in this day) is assigned to a shift. The selected team is assigned by the manager to the building with the highest needs. All the preferences (two consecutive days off, having at least one break between two shifts, etc.) are suspended if infeasibility is detected.

Example 2. In September, an unpredictable dangerous event occurs at day 8, and lasts for five days (days 8-12). The proposed adaptation of the current optimal schedule is performed by adding the teams to shifts as follows.

1. $d_{8}: S_{1}: T_{1}-S_{2}: T_{4}$

2. $d_{9}: S_{1}: T_{1}-S_{2}: T_{6}$

3. $d_{10}: S_{1}: T_{4}-S_{2}: T_{6}$

4. $d_{11}: S_{1}: T_{2}-S_{2}: T_{4}$

5. $d_{12}: S_{1}: T_{2}-S_{2}: T_{6}$

$d_{t}: S_{1}: T_{i}-S_{2}: T_{j}$ means at day $d_{t}$ team $T_{i}$ is added and assigned to shift $S_{1}$, and team $T_{j}$ is added and assigned to shift $S_{2}$.

\subsubsection{Large Scale Event (Such as a Pandemic)}

In this case, there is a sharp increase in the demand of the physicians and their services to tackle the consequences of the pandemic. Furthermore, all the resources are required, the changing of conditions is possible, and preferences are dropped if infeasibility is detected. In this context, the existing optimal schedule is adjusted by following a strategy allowing the increase of the resource capacity. Indeed, each day, the two non-working teams are assigned to the night shift $\left(S_{2}\right)$ so that each building has its own team during day and night shifts. In addition, the shift duration could be extended from $12 \mathrm{~h}$ to 16 and may be $24 \mathrm{~h}$, according to evaluation of the manager and the physicians.

\subsection{Comparative Study with Other Existing Methods}

It is worth noting that methods dealing with the resolution of personnel scheduling in general and physician scheduling in particular are composed of two categories. The first one is the exact methods, where an optimal solution is obtained. As an example of exact methods the integer linear programming. Generally, exact methods fail to solve the studied personnel scheduling problems because of their high complexity, which is NP-Hard. In this case, a second category of methods is required. This category is composed of approximate methods which provide a near optimal solution within an acceptable computational time. Among the most used methods, the meta-heuristics as Genetic Algorithm $(G A)$ and Simulated Annealing $(S A)$.

In this subsection, the exact proposed procedure (OPT) is compared to GA [36] and $S A$ [37]. For that aim, the $G A$ and $S A$ are tested over the test problems provided in Section 5.1. Seeking simplicity, the corresponding results are presented over Tables 9-12. Tables 9 and 10 present the detailed schedules respectively for month September and October, while applying the $G A$ and $S A$.

Tables 11 and 12 display the analysis of the results provided in Table 9.

A comparative study is carried out between $O P T, G A$, and $S A$ for September (least physicians number). The analysis of the comparative study results, are performed using: PRO: Percent Reduction of Overtime compared to the manual method, PRU: Percent Reduction of Under-load (compared to the manual method), ADO: Average Days Off, and ANS: Average Number of Night shifts. The results of this comparative study are presented in Table 13.

Based on Table 13, the $P R O$ as well as the $P R U$ are significantly greater for $O P T$ than those for GA and $S A$. Indeed, $O P T$ presents a $50 \%$ as a percent reduction of overtime load compared to the manual 
procedure. This significantly exceeds the exhibited $P R O$ of $G A$ and $S A$, which are respectively $39.58 \%$ and $37.50 \%$. Furthermore, the percent reduction of the under-load $P R U$ is reaching its maximum 100\% with the OPT procedure, where the under-load (compared to the manual procedure) is eliminated. In addition, the $P R U$ is significantly reduced while applying the $G A$ and $S A$ procedures, where the respective values are $79.17 \%$ and $75 \%$. Clearly, the displayed $P R U$ over the $O P T$ is significantly far from those presented by $G A$ and $S A$ procedures.

The other parameters ( $A D O$ and $A N S$ ) are almost the same for the three $O P T, G A$, and $S A$ methods. This provides a strong evidence that $O P T$ is clearly outperforming the other methods ( $G A$ and $S A$ ).

Table 9. Genetic Algorithm (GA) and Simulated Annealing (SA) physician schedule for September.

\begin{tabular}{|c|c|c|c|c|c|c|c|c|c|c|c|c|}
\hline \multirow[b]{3}{*}{ day } & \multicolumn{6}{|c|}{ GA } & \multicolumn{6}{|c|}{ SA } \\
\hline & \multicolumn{2}{|c|}{$\mathbf{B}_{1}$} & \multicolumn{2}{|c|}{$\mathbf{B}_{2}$} & \multicolumn{2}{|c|}{$\mathbf{B}_{3}$} & \multicolumn{2}{|c|}{$\mathbf{B}_{1}$} & \multicolumn{2}{|c|}{$\mathbf{B}_{2}$} & \multicolumn{2}{|c|}{$\mathbf{B}_{3}$} \\
\hline & $S_{1}$ & $S_{2}$ & $S_{1}$ & $S_{2}$ & $S_{1}$ & $S_{2}$ & $S_{1}$ & $S_{2}$ & $S_{1}$ & $S_{2}$ & $S_{1}$ & $S_{2}$ \\
\hline 1 & $T_{5}$ & $T_{6}$ & $T_{4}$ & $T_{6}$ & $T_{1}$ & $T_{6}$ & $T_{2}$ & $T_{5}$ & $T_{1}$ & $T_{5}$ & $T_{3}$ & $T_{5}$ \\
\hline 2 & $T_{5}$ & $T_{6}$ & $T_{3}$ & $T_{6}$ & $T_{1}$ & $T_{6}$ & $T_{2}$ & $T_{5}$ & $T_{1}$ & $T_{5}$ & $T_{3}$ & $T_{5}$ \\
\hline 3 & $T_{5}$ & $T_{6}$ & $T_{3}$ & $T_{6}$ & $T_{1}$ & $T_{6}$ & $T_{2}$ & $T_{6}$ & $T_{1}$ & $T_{6}$ & $T_{3}$ & $T_{6}$ \\
\hline 4 & $T_{5}$ & $T_{6}$ & $T_{3}$ & $T_{6}$ & $T_{2}$ & $T_{6}$ & $T_{2}$ & $T_{6}$ & $T_{4}$ & $T_{6}$ & $T_{3}$ & $T_{6}$ \\
\hline 5 & $T_{5}$ & $T_{6}$ & $T_{3}$ & $T_{6}$ & $T_{4}$ & $T_{6}$ & $T_{2}$ & $T_{6}$ & $T_{4}$ & $T_{6}$ & $T_{5}$ & $T_{6}$ \\
\hline 6 & $T_{1}$ & $T_{1}$ & $T_{2}$ & $T_{1}$ & $T_{4}$ & $T_{1}$ & $T_{1}$ & $T_{1}$ & $T_{4}$ & $T_{1}$ & $T_{5}$ & $T_{1}$ \\
\hline 7 & $T_{2}$ & $T_{2}$ & $T_{1}$ & $T_{2}$ & $T_{4}$ & $T_{2}$ & $T_{3}$ & $T_{3}$ & $T_{4}$ & $T_{3}$ & $T_{5}$ & $T_{3}$ \\
\hline 8 & $T_{3}$ & $T_{2}$ & $T_{6}$ & $T_{2}$ & $T_{1}$ & $T_{2}$ & $T_{1}$ & $T_{3}$ & $T_{6}$ & $T_{3}$ & $T_{2}$ & $T_{3}$ \\
\hline 9 & $T_{3}$ & $T_{4}$ & $T_{6}$ & $T_{4}$ & $T_{1}$ & $T_{4}$ & $T_{1}$ & $T_{3}$ & $T_{6}$ & $T_{3}$ & $T_{2}$ & $T_{3}$ \\
\hline 10 & $T_{3}$ & $T_{4}$ & $T_{6}$ & $T_{4}$ & $T_{1}$ & $T_{4}$ & $T_{1}$ & $T_{3}$ & $T_{6}$ & $T_{3}$ & $T_{2}$ & $T_{3}$ \\
\hline 11 & $T_{3}$ & $T_{4}$ & $T_{6}$ & $T_{4}$ & $T_{2}$ & $T_{4}$ & $T_{1}$ & $T_{6}$ & $T_{5}$ & $T_{6}$ & $T_{2}$ & $T_{6}$ \\
\hline 12 & $T_{3}$ & $T_{4}$ & $T_{5}$ & $T_{4}$ & $T_{2}$ & $T_{4}$ & $T_{1}$ & $T_{6}$ & $T_{5}$ & $T_{6}$ & $T_{4}$ & $T_{6}$ \\
\hline 13 & $T_{1}$ & $T_{1}$ & $T_{5}$ & $T_{1}$ & $T_{2}$ & $T_{1}$ & $T_{3}$ & $T_{3}$ & $T_{5}$ & $T_{3}$ & $T_{4}$ & $T_{3}$ \\
\hline 14 & $T_{2}$ & $T_{2}$ & $T_{5}$ & $T_{2}$ & $T_{6}$ & $T_{2}$ & $T_{2}$ & $T_{2}$ & $T_{5}$ & $T_{2}$ & $T_{4}$ & $T_{2}$ \\
\hline 15 & $T_{4}$ & $T_{3}$ & $T_{1}$ & $T_{3}$ & $T_{6}$ & $T_{3}$ & $T_{3}$ & $T_{1}$ & $T_{4}$ & $T_{1}$ & $T_{6}$ & $T_{1}$ \\
\hline 16 & $T_{4}$ & $T_{3}$ & $T_{5}$ & $T_{3}$ & $T_{1}$ & $T_{3}$ & $T_{3}$ & $T_{1}$ & $T_{4}$ & $T_{1}$ & $T_{6}$ & $T_{1}$ \\
\hline 17 & $T_{4}$ & $T_{3}$ & $T_{5}$ & $T_{3}$ & $T_{2}$ & $T_{3}$ & $T_{3}$ & $T_{2}$ & $T_{1}$ & $T_{2}$ & $T_{6}$ & $T_{2}$ \\
\hline 18 & $T_{4}$ & $T_{3}$ & $T_{5}$ & $T_{3}$ & $T_{2}$ & $T_{3}$ & $T_{3}$ & $T_{2}$ & $T_{5}$ & $T_{2}$ & $T_{1}$ & $T_{2}$ \\
\hline 19 & $T_{4}$ & $T_{3}$ & $T_{1}$ & $T_{3}$ & $T_{2}$ & $T_{3}$ & $T_{3}$ & $T_{4}$ & $T_{5}$ & $T_{4}$ & $T_{6}$ & $T_{4}$ \\
\hline 20 & $T_{1}$ & $T_{1}$ & $T_{6}$ & $T_{1}$ & $T_{2}$ & $T_{1}$ & $T_{2}$ & $T_{2}$ & $T_{5}$ & $T_{2}$ & $T_{4}$ & $T_{2}$ \\
\hline 21 & $T_{2}$ & $T_{2}$ & $T_{5}$ & $T_{2}$ & $T_{1}$ & $T_{2}$ & $T_{1}$ & $T_{1}$ & $T_{4}$ & $T_{1}$ & $T_{3}$ & $T_{1}$ \\
\hline 22 & $T_{1}$ & $T_{5}$ & $T_{3}$ & $T_{5}$ & $T_{4}$ & $T_{5}$ & $T_{5}$ & $T_{1}$ & $T_{3}$ & $T_{1}$ & $T_{2}$ & $T_{1}$ \\
\hline 23 & $T_{6}$ & $T_{5}$ & $T_{3}$ & $T_{5}$ & $T_{4}$ & $T_{5}$ & $T_{5}$ & $T_{1}$ & $T_{3}$ & $T_{1}$ & $T_{2}$ & $T_{1}$ \\
\hline 24 & $T_{6}$ & $T_{2}$ & $T_{5}$ & $T_{2}$ & $T_{4}$ & $T_{2}$ & $T_{5}$ & $T_{4}$ & $T_{3}$ & $T_{4}$ & $T_{2}$ & $T_{4}$ \\
\hline 25 & $T_{6}$ & $T_{2}$ & $T_{1}$ & $T_{2}$ & $T_{4}$ & $T_{2}$ & $T_{5}$ & $T_{2}$ & $T_{3}$ & $T_{2}$ & $T_{6}$ & $T_{2}$ \\
\hline 26 & $T_{6}$ & $T_{2}$ & $T_{1}$ & $T_{2}$ & $T_{4}$ & $T_{2}$ & $T_{5}$ & $T_{6}$ & $T_{4}$ & $T_{6}$ & $T_{1}$ & $T_{6}$ \\
\hline 27 & $T_{1}$ & $T_{1}$ & $T_{5}$ & $T_{1}$ & $T_{3}$ & $T_{1}$ & $T_{4}$ & $T_{4}$ & $T_{1}$ & $T_{4}$ & $T_{6}$ & $T_{4}$ \\
\hline 28 & $T_{2}$ & $T_{2}$ & $T_{5}$ & $T_{2}$ & $T_{3}$ & $T_{2}$ & $T_{6}$ & $T_{6}$ & $T_{1}$ & $T_{6}$ & $T_{2}$ & $T_{6}$ \\
\hline
\end{tabular}


Table 10. GA and SA physician schedule for October.

\begin{tabular}{|c|c|c|c|c|c|c|c|c|c|c|c|c|}
\hline \multirow[b]{3}{*}{ day } & \multicolumn{6}{|c|}{ GA } & \multicolumn{6}{|c|}{ SA } \\
\hline & \multicolumn{2}{|c|}{$\mathbf{B}_{1}$} & \multicolumn{2}{|c|}{$\mathbf{B}_{2}$} & \multicolumn{2}{|c|}{$\mathbf{B}_{3}$} & \multicolumn{2}{|c|}{$\mathbf{B}_{1}$} & \multicolumn{2}{|c|}{$\mathbf{B}_{2}$} & \multicolumn{2}{|c|}{$\mathbf{B}_{3}$} \\
\hline & $S_{1}$ & $S_{2}$ & $S_{1}$ & $S_{2}$ & $S_{1}$ & $S_{2}$ & $S_{1}$ & $S_{2}$ & $S_{1}$ & $S_{2}$ & $S_{1}$ & $S_{2}$ \\
\hline 1 & $T_{6}$ & $T_{3}$ & $T_{5}$ & $T_{3}$ & $T_{2}$ & $T_{3}$ & $T_{4}$ & $T_{1}$ & $T_{5}$ & $T_{1}$ & $T_{6}$ & $T_{1}$ \\
\hline 2 & $T_{6}$ & $T_{4}$ & $T_{5}$ & $T_{4}$ & $T_{2}$ & $T_{4}$ & $T_{4}$ & $T_{3}$ & $T_{5}$ & $T_{3}$ & $T_{6}$ & $T_{3}$ \\
\hline 3 & $T_{6}$ & $T_{1}$ & $T_{5}$ & $T_{1}$ & $T_{2}$ & $T_{1}$ & $T_{4}$ & $T_{2}$ & $T_{5}$ & $T_{2}$ & $T_{6}$ & $T_{2}$ \\
\hline 4 & $T_{6}$ & $T_{5}$ & $T_{3}$ & $T_{5}$ & $T_{1}$ & $T_{5}$ & $T_{4}$ & $T_{2}$ & $T_{1}$ & $T_{2}$ & $T_{6}$ & $T_{2}$ \\
\hline 5 & $T_{6}$ & $T_{5}$ & $T_{3}$ & $T_{5}$ & $T_{1}$ & $T_{5}$ & $T_{4}$ & $T_{2}$ & $T_{1}$ & $T_{2}$ & $T_{6}$ & $T_{2}$ \\
\hline 6 & $T_{4}$ & $T_{4}$ & $T_{3}$ & $T_{4}$ & $T_{1}$ & $T_{4}$ & $T_{2}$ & $T_{2}$ & $T_{1}$ & $T_{2}$ & $T_{5}$ & $T_{2}$ \\
\hline 7 & $T_{3}$ & $T_{3}$ & $T_{4}$ & $T_{3}$ & $T_{2}$ & $T_{3}$ & $T_{3}$ & $T_{3}$ & $T_{1}$ & $T_{3}$ & $T_{5}$ & $T_{3}$ \\
\hline 8 & $T_{1}$ & $T_{3}$ & $T_{4}$ & $T_{3}$ & $T_{2}$ & $T_{3}$ & $T_{6}$ & $T_{3}$ & $T_{4}$ & $T_{3}$ & $T_{2}$ & $T_{3}$ \\
\hline 9 & $T_{1}$ & $T_{3}$ & $T_{4}$ & $T_{3}$ & $T_{2}$ & $T_{3}$ & $T_{6}$ & $T_{3}$ & $T_{4}$ & $T_{3}$ & $T_{2}$ & $T_{3}$ \\
\hline 10 & $T_{1}$ & $T_{4}$ & $T_{6}$ & $T_{4}$ & $T_{5}$ & $T_{4}$ & $T_{6}$ & $T_{5}$ & $T_{4}$ & $T_{5}$ & $T_{2}$ & $T_{5}$ \\
\hline 11 & $T_{1}$ & $T_{4}$ & $T_{6}$ & $T_{4}$ & $T_{5}$ & $T_{4}$ & $T_{6}$ & $T_{2}$ & $T_{5}$ & $T_{2}$ & $T_{3}$ & $T_{2}$ \\
\hline 12 & $T_{1}$ & $T_{4}$ & $T_{6}$ & $T_{4}$ & $T_{5}$ & $T_{4}$ & $T_{6}$ & $T_{2}$ & $T_{5}$ & $T_{2}$ & $T_{3}$ & $T_{2}$ \\
\hline 13 & $T_{3}$ & $T_{3}$ & $T_{6}$ & $T_{3}$ & $T_{5}$ & $T_{3}$ & $T_{4}$ & $T_{4}$ & $T_{5}$ & $T_{4}$ & $T_{3}$ & $T_{4}$ \\
\hline 14 & $T_{6}$ & $T_{1}$ & $T_{2}$ & $T_{1}$ & $T_{5}$ & $T_{1}$ & $T_{1}$ & $T_{1}$ & $T_{5}$ & $T_{1}$ & $T_{3}$ & $T_{1}$ \\
\hline 15 & $T_{2}$ & $T_{1}$ & $T_{4}$ & $T_{1}$ & $T_{3}$ & $T_{1}$ & $T_{2}$ & $T_{5}$ & $T_{1}$ & $T_{5}$ & $T_{6}$ & $T_{5}$ \\
\hline 16 & $T_{2}$ & $T_{1}$ & $T_{4}$ & $T_{1}$ & $T_{3}$ & $T_{1}$ & $T_{2}$ & $T_{5}$ & $T_{1}$ & $T_{5}$ & $T_{6}$ & $T_{5}$ \\
\hline 17 & $T_{2}$ & $T_{1}$ & $T_{4}$ & $T_{1}$ & $T_{3}$ & $T_{1}$ & $T_{2}$ & $T_{4}$ & $T_{1}$ & $T_{4}$ & $T_{6}$ & $T_{4}$ \\
\hline 18 & $T_{2}$ & $T_{5}$ & $T_{4}$ & $T_{5}$ & $T_{3}$ & $T_{5}$ & $T_{2}$ & $T_{4}$ & $T_{1}$ & $T_{4}$ & $T_{6}$ & $T_{4}$ \\
\hline 19 & $T_{2}$ & $T_{5}$ & $T_{4}$ & $T_{5}$ & $T_{3}$ & $T_{5}$ & $T_{2}$ & $T_{4}$ & $T_{5}$ & $T_{4}$ & $T_{3}$ & $T_{4}$ \\
\hline 20 & $T_{1}$ & $T_{1}$ & $T_{6}$ & $T_{1}$ & $T_{5}$ & $T_{1}$ & $T_{1}$ & $T_{1}$ & $T_{5}$ & $T_{1}$ & $T_{3}$ & $T_{1}$ \\
\hline 21 & $T_{5}$ & $T_{5}$ & $T_{6}$ & $T_{5}$ & $T_{4}$ & $T_{5}$ & $T_{6}$ & $T_{6}$ & $T_{5}$ & $T_{6}$ & $T_{3}$ & $T_{6}$ \\
\hline 22 & $T_{4}$ & $T_{6}$ & $T_{5}$ & $T_{6}$ & $T_{2}$ & $T_{6}$ & $T_{3}$ & $T_{2}$ & $T_{5}$ & $T_{2}$ & $T_{1}$ & $T_{2}$ \\
\hline 23 & $T_{4}$ & $T_{3}$ & $T_{5}$ & $T_{3}$ & $T_{2}$ & $T_{3}$ & $T_{3}$ & $T_{2}$ & $T_{4}$ & $T_{2}$ & $T_{1}$ & $T_{2}$ \\
\hline 24 & $T_{4}$ & $T_{3}$ & $T_{5}$ & $T_{3}$ & $T_{2}$ & $T_{3}$ & $T_{3}$ & $T_{2}$ & $T_{4}$ & $T_{2}$ & $T_{1}$ & $T_{2}$ \\
\hline 25 & $T_{4}$ & $T_{1}$ & $T_{5}$ & $T_{1}$ & $T_{6}$ & $T_{1}$ & $T_{3}$ & $T_{2}$ & $T_{4}$ & $T_{2}$ & $T_{6}$ & $T_{2}$ \\
\hline 26 & $\mathrm{~T}_{4}$ & $T_{1}$ & $T_{5}$ & $T_{1}$ & $T_{6}$ & $T_{1}$ & $T_{3}$ & $T_{2}$ & $T_{5}$ & $T_{2}$ & $T_{6}$ & $T_{2}$ \\
\hline 27 & $T_{2}$ & $T_{2}$ & $T_{3}$ & $T_{2}$ & $T_{6}$ & $T_{2}$ & $T_{1}$ & $T_{1}$ & $T_{5}$ & $T_{1}$ & $T_{6}$ & $T_{1}$ \\
\hline 28 & $T_{3}$ & $T_{3}$ & $T_{6}$ & $T_{3}$ & $T_{2}$ & $T_{3}$ & $T_{4}$ & $T_{4}$ & $T_{5}$ & $T_{4}$ & $T_{6}$ & $T_{4}$ \\
\hline
\end{tabular}


Table 11. Analysis of the GA and SA physician schedule for September.

\begin{tabular}{cccccccc}
\hline & \multicolumn{3}{c}{$G A$} & \multicolumn{3}{c}{$S A$} \\
\hline Team & $N P_{m}$ & $N S_{m}$ & $O T_{m}$ & $U L_{m}$ & $N S_{m}$ & $O T_{m}$ & $U L_{m}$ \\
\hline$T_{1}$ & 3 & 22 & 168 & 0 & 21 & 132 & 0 \\
\hline$T_{2}$ & 3 & 21 & 132 & 0 & 20 & 96 & 0 \\
\hline$T_{3}$ & 3 & 18 & 24 & 0 & 21 & 132 & 0 \\
\hline$T_{4}$ & 3 & 18 & 24 & 0 & 16 & 0 & -48 \\
\hline$T_{5}$ & 3 & 17 & 0 & -12 & 17 & 0 & -12 \\
\hline$T_{6}$ & 3 & 16 & 0 & -48 & 17 & 0 & -12 \\
\hline & & Total & 348 & -60 & & 360 & -72 \\
\hline
\end{tabular}

Table 12. Detailed analysis of the GA and SA physician schedule for September.

\begin{tabular}{|c|c|c|c|c|c|c|c|c|c|c|}
\hline \multirow[b]{2}{*}{ Team } & \multicolumn{5}{|c|}{$G A$} & \multicolumn{5}{|c|}{$S A$} \\
\hline & $N S_{m}$ & $N N S_{m}$ & $N D O_{m}$ & $M D O_{m}$ & $m D O_{m}$ & $N S_{m}$ & $N N S_{m}$ & $N D O_{m}$ & $M D O_{m}$ & $m D O_{m}$ \\
\hline$T_{1}$ & 22 & 4 & 10 & 2 & 1 & 21 & 6 & 9 & 2 & 1 \\
\hline$T_{2}$ & 21 & 8 & 11 & 3 & 1 & 20 & 5 & 10 & 2 & 1 \\
\hline$T_{3}$ & 18 & 5 & 10 & 3 & 1 & 21 & 5 & 9 & 3 & 1 \\
\hline$T_{4}$ & 18 & 4 & 10 & 3 & 1 & 16 & 3 & 13 & 4 & 1 \\
\hline$T_{5}$ & 17 & 2 & 11 & 6 & 1 & 17 & 2 & 11 & 3 & 1 \\
\hline$T_{6}$ & 16 & 5 & 12 & 4 & 2 & 17 & 7 & 11 & 2 & 1 \\
\hline
\end{tabular}

Table 13. Comparison of OPT, GA, and SA for September.

\begin{tabular}{ccccc}
\hline & PRO & PRU & ADO & ANS \\
\hline OPT & 50.00 & 100 & 10.67 & 4.67 \\
\hline$G A$ & 39.58 & 79.17 & 10.67 & 4.67 \\
\hline$S A$ & 37.50 & 75 & 10.50 & 4.67 \\
\hline
\end{tabular}

\section{Conclusions}

In this paper, a case study of physician scheduling problem in an ICU is addressed. In this ICU, the physician schedule is a monthly one. A team of schedulers used to manually prepare this schedule. This team spends more than two weeks for the preparation of such a schedule. This is because of the hardness and the complexity of this procedure. In this context, new non-classical constraints are included. The manual schedules are presenting several drawbacks. Indeed, the workload is unbalanced and the overtime is excessive. In addition, several physicians' preferences are not satisfied (two consecutive days off per week for example).

In order to overcome these drawbacks, the problem is modeled as an integer linear program (ILP). In this ILP, all the already mentioned requirements and preferences are taken into account. The collected data are for two months, this might not be allowing to reach accurate conclusions. However, one of the studied months is with the least number of physicians (resources), which allows studying the proposed procedure in a critical situation. 
The experimental results are satisfactory and an important reduction of the overtime which is up to $69.23 \%$ is obtained. In addition, the unbalanced workload is significantly reduced. Furthermore, the under-load is completely eliminated. These results encourage the scheduler to implement the optimized procedure.

The automation (optimized) is not a time consuming procedure, since the total time to get the schedule is not exceeding two hours instead of more than two weeks for the manual procedure. In addition, the proposed procedure is enriched with other features which permit to deal with unpredictable events ranging from simple one (absence of a physician) to a major event (as pandemic), where conditions are changing. These features are transforming the obtained optimal schedules (in normal situation) into new ones with simple transformations. These transformations are performed by the manager according to the situations. The added part is allowing more adaptability and flexibility of the proposed procedures.

In future works, other objective functions will be experienced as minimizing the total unbalanced workload, and other preferences and requirements will be introduced. These requirements and preferences are conflicting which will be treated over a goal programming model.

Author Contributions: The contributions of authors are as follows: Conceptualization, L.H.; methodology, L.H.; software, A.G.; validation, L.H., A.G. and M.M.M.; data curation, L.H., A.G. and M.M.M.; writing original draft preparation, L.H. All authors have read and agreed to the published version of the manuscript.

Funding: Deputyship for Research \& Innovation, "Ministry of Education" in Saudi Arabia grant number IFKSUHI-1439-001.

Acknowledgments: The authors extend their appreciation to the Deputyship for Research \& Innovation, "Ministry of Education" in Saudi Arabia for funding this research work through the project number IFKSUHI-1439-001.

Conflicts of Interest: The authors declare no conflict of interest.

\section{References}

1. Blum, K.; Löffert, S.; Offermanns, M.; Perner, P. Krankenhaus Barometer-Umfrage 2013; Düsseldorf Technical Report; Deutsches Krankenhaus Institut e.V.: Düsseldorf Germany, 2013.

2. Bölt, U. Statistische Krankenhausdaten: Grundund Kostendaten der Krankenhäuser 2011. In KrankenhausReport 2014: Schwerpunkt: Patientensicherheit; Klauber, J., Geraedts, M., Friedrich, J., Wasem, J., Eds.; Schattauer: Stuttgart, Germany, 2014; pp. 293-328.

3. Aykin, T. A comparative evaluation of modeling approaches to the labor shift scheduling problem. Eur. J. Oper. 2000, 125, 381-397. [CrossRef]

4. Kuo, Y.H. Integrating simulation with simulated annealing for scheduling physicians in an understaffed emergency department. HKIE Trans. 2014, 21, 253-261. [CrossRef]

5. Zeltyn, S.; Marmor, Y.N.; Mandelbaum, A.; Carmeli, B.; Greenshpan, O.; Mesika, Y. Simulation-based models of emergency departments: Operational, tactical and strategic staffing. ACM Trans. Model. Comput. Simul. 2011, 21, 24. [CrossRef]

6. Knauth, P. The design of shift systems. Ergonomics 1993, 36, 15-28. [CrossRef] [PubMed]

7. Knauth, P. Design better shift systems. Appl. Ergon. 1996, 27, 39-44. [CrossRef]

8. Grantcharov, T.; Bardram, L.; Peter, F.; Rosenberg, J. Laparoscopic performance after one night on call in a surgical department: Prospective study. Br. Med J. 2001, 323, 1222-1223. [CrossRef]

9. Santos, M.; Eriksson, H. Insights into physician scheduling: A case study of public hospital departments in Sweden. Int. J. Health Care Qual. Assur. 2014, 27, 76-90.

10. Bodenheimer, T.S.; Smith, M.D. Primary care: Proposed solutions to the physician shortage without training more physicians. Health Aff. 2013, 32, 1881-1886. [CrossRef]

11. McManus, I.C.; Smithers, E.; Partridge, P.; Keeling, A.; Fleming, A.P.R. A levels and intelligence as predictors of medical careers in UK doctors: 20 year prospective study. Br. Med. J. 2003, 327, 139-142. [CrossRef]

12. Venkat, A.; Kekre, S.; Hegde, G.G.; Shang, J.; Campbell, T.P. Strategic management of operations in the emergency department. Prod. Oper. Manag. 2015, 24, 1706-1723. [CrossRef] 
13. Fügener, A.; Schiffels, S.; Kolisch, R. Overutilization and underutilization of operating rooms-insights from behavioral health care operations management. Health Care Manag. 2017, 20, 115-128. [CrossRef]

14. Ernst, A.T.; Jiang, H.; Krishnamoorthy, M.; Sier, D. Staff scheduling and rostering: A review of applications, methods and models. Eur. J. Oper. Res. 2004, 153, 3-27. [CrossRef]

15. Ernst, A.T.; Jiang, H.; Krishnamoorthy, M.; Owens, B.; Sier, D. An annotated bibliography of personnel scheduling and rostering. Ann. Oper. Res. 2004, 127, 21-144. [CrossRef]

16. den Bergh, J.V.; Beliën, J.; Bruecker, P.D.; Demeulemeester, E.; Boeck, L.D. Personnel scheduling: A literature review. Eur. J. Oper. Res. 2013, 226, 367-385. [CrossRef]

17. Bruecker, P.D.; den Bergh, J.V.; Beliën, J.; Demeulemeester, E. Workforce planning incorporating skills: State of the art. Eur. J. Oper. Res. 2015, 243, 1-16. [CrossRef]

18. Burke, E.K.; Causmaecker, P.D.; Berghe, G.C.; Landeghem, H. The state of the art of nurse rostering. J. Sched. 2004, 7, 441-499. [CrossRef]

19. Cheang, B.; Li, H.; Lim, A.; Rodrigues, B. Nurse rostering problems-A bibliographic survey. Eur. J. Oper. Res. 2003, 151, 447-460. [CrossRef]

20. Hayes, L.J.; O’Brien-Pallas, L.; Duffield, C.; Shamian, J.; Buchan, J.; Hughes, F. Nurse turnover: A literature review-An update. Int. J. Nurs. Stud. 2012, 49, 887-905. [CrossRef]

21. Nosbusch, J.M.; Weiss, M.E.; Bobay, K.L. An integrated review of the literature on challenges confronting the acute care staff-nurse in discharge planning. J. Clin. Nurs. 2011, 20, 754-774. [CrossRef]

22. Cardoen, B.; Demeulemeester, E.; Beliën, J. Operating room planning and scheduling: A literature review. Eur. J.Oper. Res. 2010, 201, 921-932. [CrossRef]

23. Erhard, M.; Schoenfelder, J.; Fügener, A.; Brunner, J.O. State of the art in physician scheduling. Eur. J. Oper. Res. 2018, 265, 1-18. [CrossRef]

24. Kurt, P.D.; Zhang, M.; Marentay, B.; Govind, N. Improving physician schedules by leveraging equalization: Cases from hospitals in U.S. Omega to be published. [CrossRef]

25. De Vrugt, N.M.V.; Luen-English, S.T.; Bastiaansen, W.A.P.; Kleinluchtenbeld, S.; Lardinois, W.T.P.; Pots, M.H.; Schoonbergen, D.J.; Hans, E.W.; Hurink, J.L.; Boucherie, R.J. Integrated scheduling of tasks and gynecologists to improve patient appointment scheduling; a case study. Oper. Res. Health Care 2018, 16, 10-19. [CrossRef]

26. Wong, T.; Tsai, M.H.; Urman, R.D. The expansion of non-operating room anesthesia services. Perioper. Care Oper. Room Manag. 2017, 9, 39-42. [CrossRef]

27. Thielen, C. Duty rostering for physicians at a department of orthopedics and trauma surgery. Oper. Res. Health Care 2018, 19, 80-91. [CrossRef]

28. Dowell, J.D.; Makary, M.S.; Brocone, M.; Sarbinoff, J.G.; Vargas, I.G.; Gadkari, M. Lean Six Sigma Approach to Improving Interventional Radiology Scheduling. J. Am. Coll. Radiol. 2017, 14, 1316-1321. [CrossRef] [PubMed]

29. Hong, Y.C.; Cohn, A.; Epelman, M.A.; Alpert, A. Creating resident shift schedules under multiple objectives by generating and evaluating the Pareto frontier. Oper. Res. Ealth Care to be published. [CrossRef]

30. Zaerpour, F.; Bischak, D.P.; Menezes, M.B.C. Coordinated lab-clinics: A tactical assignment problem in healthcare. Eur. J. Oper. Res. 2017, 263, 283-294. [CrossRef]

31. Volland, J.; Fügener, A.; Brunner, J.O. A column generation approach for the integrated shift and task scheduling problem of logistics assistants in hospitals. Eur. J. Oper. Res. 2017, 260, 316-334. [CrossRef]

32. Huang, W.T.; Chen, P.S.; Liu, J.J.; Chen, Y.R.; Chen, Y.H. Dynamic configuration scheduling problem for stochastic medical resources. J. Biomed. Inform. 2018, 80, 96-105. [CrossRef]

33. Gross, C.N.; Brunner, J.O.; Blobner, M. Hospital physicians can't get no long-term satisfaction- An indicator for fairness in preference fulfillment on duty schedules. Health Care Manag. Sci. to be published. [CrossRef] [PubMed]

34. Niroumandrad, N.; Lahrichi, N. A stochastic tabu search algorithm to align physician schedule with patient flow. Health Care Manag. Sci. 2018, 21, 244-258. [CrossRef]

35. Gross, C.N.; Fügener, A.; Brunner, J.O. Online rescheduling of physicians in hospitals. Flex Serv. Manuf. J. 2018, 30, 296-328. [CrossRef] 
36. Puente, J.; Gómez, A.; Fernández, I.; Priore, P. Medical doctor rostering problem in a hospital emergency department by means of genetic algorithms. Comput. Ind. Eng. 2009, 56, 1232-1242. [CrossRef]

37. Rosocha, L.; Vernerova, S.; Verner, R. Medical staff scheduling using simulated annealing. Qual. Innov. Prosper. 2014, 19, 1-8. [CrossRef]

Publisher's Note: MDPI stays neutral with regard to jurisdictional claims in published maps and institutional affiliations.

(c) 2020 by the authors. Licensee MDPI, Basel, Switzerland. This article is an open access article distributed under the terms and conditions of the Creative Commons Attribution (CC BY) license (http:// creativecommons.org/licenses/by/4.0/). 